\title{
Singularities in the complex physical plane for deep water waves
}

\author{
Gregory R. Baker† and Chao Xie \\ Department of Mathematics, The Ohio State University, 231 West 18th Avenue, Columbus, OH 43210, \\ USA
}

(Received 10 September 2010; revised 27 March 2011; accepted 30 June 2011; first published online 22 September 2011)

Deep water waves in two-dimensional flow can have curvature singularities on the surface profile; for example, the limiting Stokes wave has a corner of $2 \pi / 3$ radians and the limiting standing wave momentarily forms a corner of $\pi / 2$ radians. Much less is known about the possible formation of curvature singularities in general. A novel way of exploring this possibility is to consider the curvature as a complex function of the complex arclength variable and to seek the existence and nature of any singularities in the complex arclength plane. Highly accurate boundary integral methods produce a Fourier spectrum of the curvature that allows the identification of the nearest singularity to the real axis of the complex arclength plane. This singularity is in general a pole singularity that moves about the complex arclength plane. It approaches the real axis very closely when waves break and is associated with the high curvature at the tip of the breaking wave. The behaviour of these singularities is more complex for standing waves, where two singularities can be identified that may collide and separate. One of them approaches the real axis very closely when a standing wave forms a very narrow collapsing column of water almost under free fall. In studies so far, no singularity reaches the real axis in finite time. On the other hand, the surface elevation $y(x)$ has square-root singularities in the complex $x$ plane that do reach the real axis in finite time, the moment when a wave first starts to break. These singularities have a profound effect on the wave spectra.

Key words: waves/free-surface flows

\section{Introduction}

Detailed knowledge of the behaviour of water waves has immediate and important applications in a variety of fields, notably climatology, marine transport and coastal environments. What makes detailed studies of water waves difficult is the nature of the nonlinear surface conditions. Progress, then, has often relied on simplifications to the nonlinear behaviour in a variety of techniques, in particular simple linearization, amplitude expansions or weakly nonlinear theories. Recently, there have been new 
developments in mathematical theory of the full nonlinear motion based on modern techniques in functional analysis; see, for example, Craig \& Wayne (2007).

The challenge in rigorous mathematical analysis is the possibility of waves breaking or forming singularities in slope or curvature in finite time. Two striking examples confirm that the possibility is real. Toland (1996) proves that the limiting Stokes wave has a corner of angle $2 \pi / 3$ radians that retains its form as the wave propagates. Okamura (1998) proves that the limiting standing waves reaches a crest with a corner of $\pi / 2$ radians momentarily before subsiding with the disappearance of the corner until the next event. Consequently, long time existence in Sobolev spaces is difficult to establish without restrictions on the initial data that exclude these possibilities. Nevertheless, some progress has been made. Existence and uniqueness of solutions have been proven locally in time if the initial conditions are analytic by Shinbrot (1976) and Kano \& Nishida (1979). Recently, new results by Wu (1997, 1999) prove existence and uniqueness in certain Sobolev spaces for both two- and threedimensional motion for finite time, provided that the initial wave surface does not self-intersect or that the initial motion satisfies the Taylor condition, that is, the surface does not accelerate faster than gravity in the inward direction normal to the water surface. One of the results we report is that the Taylor condition is satisfied even after the waves have broken.

Provided the initial data are small enough, long time existence and uniqueness has been established for two-dimensional motion by Wu (2009) and global existence and uniqueness in time has been established for three-dimensional motion by Germain, Masmoudi \& Shatah (2009). All these results hold under the assumption that the surface returns to a flat surface in the far field, but it seems plausible that similar results will hold for periodic domains, a case that is more practical in ocean studies.

While general rigorous theory establishes the mathematical context in which water waves appear, it is often specific generic solutions that shed insight. Progress has been made here too, based on numerical simulations and new asymptotic approaches. In particular, a new conceptual paradigm has emerged from the pioneering work of Moore (1979) on the spontaneous appearance of curvature singularities on vortex sheets. A vortex sheet may be used to represent the interface between two immiscible, incompressible fluids in the limit of zero viscosity. Moore's study considers the densities of the two fluids to be comparable and is restricted to two-dimensional periodic flow with an analytic initial condition. His results suggest the appearance of 3/2-branch-point singularities in the complex plane of the surface parametrization variable. Specifically, if the surface location is expressed in parametric form $(x(p, t), y(p, t))$ where $p$ is a Lagrangian variable, then $(x(p, t), y(p, t)) \sim\left(p-p_{s}\right)^{3 / 2}$ near a point $p_{s}$ in the complex plane of $p$. There may be several such points and they move about the complex plane while retaining the 3/2-power singularity in $(x(p), y(p, t))$. They can approach and reach the real axis in finite time, at which point they are curvature singularities on the vortex sheet. Note that the circulation variable $\Gamma$ may be the choice for the surface variable; then the curvature behaves as $\kappa \sim\left(\Gamma-\Gamma_{s}\right)^{-1 / 2}$, where $\Gamma_{s}$ moves in the complex circulation plane and reaches the real axis in finite time. Since curvature and circulation are real physical quantities, this singularity is not an artifact of the parameterization.

Further work has recast Moore's results as arising from the analytic continuation of the interface conditions specified along the real axis of $p$ into the complex $p$-plane. In particular, the motion of the surface may be described by boundary integrals; there are several different formulations, but the ones by Baker, Meiron \& Orszag (1980, 1982) have particular advantages for analysis and numerical methods. The 
first successes from the analytic continuation of boundary integrals for understanding singularity formation on vortex sheets came from the work of Caflisch \& Semmes (1990) and Caflisch \& Orellana (1989) among others. Analytic continuation creates complex hyperbolic partial differential equations with a remaining boundary integral that is exponentially small in the distance from the real axis. Asymptotically, then, it is easy to understand the formation of the Moore singularities far from the real axis, and Cowley, Baker \& Tanveer (1999) have developed a consistent asymptotic theory that describes the nature of the vortex sheet as a Moore singularity approaches and reaches the real axis. Direct numerical calculations of vortex sheet motion have been used by Krasny (1986) and Shelley (1992) to locate the presence of singularities in the complex plane through the nature of the decay of the Fourier series as described by Sulem, Sulem \& Frisch (1983), and the results confirm the asymptotic theories. Further work by Nie \& Baker (1998) has established that Moore singularities are present in axisymmetric vortex sheets, and by Ishihara \& Kaneda (1994) and Hou \& $\mathrm{Hu}(2003)$ in three-dimensional vortex sheets.

The knowledge gained from understanding the formation of curvature singularities on vortex sheets has led directly to understanding their formation in more general cases, specifically when the densities of the two fluids separated by a free surface are different. In particular, Baker, Caflisch \& Siegel (1993) find Moore singularities during the two-fluid Rayleigh-Taylor instability, where heavier fluid falls into lighter fluid. Vorticity is generated along the sides of the developing spikes, thus effectively creating a vortex sheet of almost uniform strength that then becomes unstable to the Kelvin-Helmholtz instability. The result is the appearance of Moore singularities that track towards and reach the real axis in finite time. The location of the formation of the curvature singularities on either side of the falling spike changes with changes in the density difference. The closer the density of the lighter fluid approaches to zero, the closer the pair of curvature singularities appear to the tip of the spike. Indeed, when the lighter fluid has zero density, the Moore singularities cancel each other leaving no apparent singularity in $(x(p), y(p))$. The same phenomenon occurs when light fluid lies above heavier fluid. In this case, the interface exhibits gravitationally stable waves and the Moore singularities stay away from the real axis when the waves do not break, but there appears to be no singularity in $(x(p), y(p))$ when the fluid above has no density.

The apparent change in the nature of the singularities when one of the fluids has no density motivated Tanveer $(1991,1993)$ to study free surface flow of a single fluid. He uses a conformal map from a periodic domain to a unit circle. If $z=x+$ iy is a complex spatial variable for a $2 \pi$-periodic domain in $x$ and $y$ is a vertical coordinate directed upwards, and $\eta$ is the variable in the circle, then the map is

$$
z(\eta, t)=2 \pi+\mathrm{i} \ln (\eta)+\mathrm{i} f(\eta, t),
$$

where $f(\eta, t)$ is analytic in a disk that contains the unit disk and so has a convergent Taylor series. Tanveer (1991) considers propagating waves of permanent form and proves that the only possible singularities in $f$ are of the square-root type. In particular,

$$
f(\eta, t)=\sum_{n=0}^{\infty} d_{n}(t)\left(\eta-\eta_{s}\right)^{n / 2},
$$

where $\eta_{s}$ lies outside the unit disk. Two such singularities merge and become a cuberoot singularity as the family of permanent waves reaches its limiting form. Thus a corner singularity appears with an angle of $2 \pi / 3$ radians. Subsequently, Tanveer (1993) 
shows how such square-root singularities arise from general initial conditions. There is no contradiction with the previous numerical results of Baker et al. (1993) because of the unknown relationship between the Lagrangian variable $p$ and the conformal mapping variable $\eta$. Indeed, while $z(p)$ may be analytic outside the unit disk, there may be singularities in the map between $p$ (as a complex variable) and $\eta$. Indeed, results reported in this article confirm the presence of such singularities and their correspondence to the square-root singularities in $f(\eta)$.

What then is the consequence of square-root singularities in $f(\eta)$ ? What is needed to answer this question is a choice of variables that is independent of parametrization, and the obvious choice is the arclength variable. We show in this article that the curvature of the surface of deep water waves contains pole singularities in the complex arclength plane, and that these poles correspond directly to the square-root singularities in the $\eta$-plane and to zeros in $z_{p}$ in the Lagrangian variable. The evidence is obtained by direct numerical simulations with exceedingly high accuracy that allows form fits to the Fourier spectra of the curvature in arclength to identify the type and location of singularities nearest to the real axis. Initially, the water surface is chosen to be analytic in the Lagrangian variable, but it contains a location where $z_{p}=0$. By constructing the conformal map from $\eta$ to $p$, we confirm that there is a corresponding initial square-root singularity in $f(\eta)$ and a pole singularity in the curvature $\kappa(s)$. These singularities are then tracked as the water surface evolves. We take two sets of initial conditions: one set is associated with travelling waves and the other set with standing waves. In both cases, the singularities are of only one type, the square-root type in the $\eta$-plane predicted by Tanveer (1993), or a pole singularity in the arclength plane. Their presence appears generic.

The evidence is also strong that the curvature pole singularities do not reach the real axis in finite time. What then is their relevance? The first observation established in this article is their connection to breaking waves. For propagating waves, the curvature singularities are associated with the wave crests where the curvature is largest. When the wave amplitudes are small, the singularities track through the complex arclength plane a certain distance above the location of the maximum curvature on the real axis. For larger amplitudes, the singularities move up and down as nonlinearity modulates the wave. For large amplitudes, the waves quickly steepen and break. Here the curvature singularities move steadily towards the real axis and approach it closely. When the wave breaks there is a noticeable slowdown in the approach to the real axis and it seems unlikely that they will reach the real axis before the tip of the breaker meets the water surface below it.

Another way to look at the potential for waves to break is to examine the wave profile $y(x, t)$ and ask whether there are singularities in the complex $x$ plane. Indeed there are and they are of the square-root type. When a wave first breaks, the slope becomes infinite. At this moment the square-root singularity has reached the real axis. There is a clear record of the singularity approaching the real axis steadily, which suggests the possibility of predicting wave breaking well in advance. Even in the absence of wave breaking, square-root singularities in $y(x)$ affect the tail of the wave spectra very strongly. Our results suggest a different approach to understanding possible statistical equilibrium of wave spectra as being the consequence of squareroot singularities travelling above the real axis as water waves propagate.

The initial condition to simulate standing waves is just a sinusoidal wave profile released from rest. The expectation was that curvature singularities would reach the real axis for waves of sufficient amplitude, forming corners as in the limiting standing wave. To our surprise, this possible behaviour is not generic. Indeed, the curvature 
singularities approach the real axis very closely, and the profile forms very narrow spikes of fluid which collapse under gravity and are re-absorbed by the water surface in a smooth way. Of course, surface tension effects will be important under these conditions, effects that we have neglected in this study.

There have been other attempts to determine the possible singularity structure of water waves. Kuznetsov, Spector \& Zakharov (1994) use the Hamiltonian formulation for water waves, but neglect gravity and expand the Hamiltonian in an approximation. Their predicted singularities do not agree with the results of this study. Fontelos \& de la Hoz (2010) use a boundary integral formulation and seek self-similar solutions that might match their numerical results. There are several undetermined parameters in the self-similar solutions which raises difficulties in matching to numerical results. This approach failed in studies of singularity formation on vortex sheets. Indeed, Cowley et al. (1999) use the presence of 3/2-power singularities in the complex plane to match with the appropriate self-similar solution. We repeat the calculation of Fontelos $\&$ de la Hoz (2010) and find pole singularities in the complex arclength plane for the curvature. Since Fontelos \& de la Hoz (2010) do not use this information in the match to the self-similar solutions, their results may be in error. We suggest, then, that it is the approach of this work, following previous work, that is best capable of understanding possible curvature singularity formation on free surfaces.

There have been successful local models for breaking waves. In Longuet-Higgins (1980), a tip of a plunging breaker is modelled as a hyperbola whose asymptotes are collapsing. The form of the solutions appears very similar to those observed in numerical simulations and in observations of actual breaking waves. We reproduce the results in Longuet-Higgins (1980) for Lagrangian motion and show clearly the consistency of the results with the presence of pole singularities in the complex arclength plane. By using a variant of the Taylor condition, a model for the inside curl of a breaking wave has been developed by Longuet-Higgins (1982) that agrees very well with observed surface profiles. This too has a direct connection to a pole singularity, different from the one near the tip and further away from the real axis of the complex arclength plane. Both these models support the view that the pole singularities do not reach the real axis in finite time.

\section{Boundary integral technique}

The motion of deep water waves is usually modelled as the motion of a sharp interface above water considered to be an incompressible, inviscid fluid. In the absence of wind shear, the motion of the air above the interface is neglected because the air density is extremely small compared to the density of water. In addition, surface tension may be neglected if the water waves have wavelengths longer than a few centimetres, an assumption made in this paper. In general, water waves propagate with a spanwise extent that is much longer than their wavelength. Thus it is reasonable to assume two-dimensional motion.

\subsection{Mathematical formulation}

Align the coordinate system so that $x$ is a horizontal coordinate in the direction of propagation of the waves and $y$ is a vertical coordinate opposite to the direction of gravity. The location of the water surface may be expressed in parametric form as a complex-valued function $z(p, t)=x(p, t)+\mathrm{i} y(p, t)$, where $p$ is a Lagrangian marker.

If the motion of the water is irrotational, a common assumption in studies of water waves, then the velocity may be expressed in terms of a velocity potential $\phi$. The 
velocity potential satisfies Laplace's equation in the region occupied by the water. Bernoulli's equation evaluated at the surface, where the pressure is a constant set to zero for convenience, provides an evolution equation for the velocity potential. All that is needed is the calculation of the normal derivative of the velocity potential to complete the specification of the velocity of the surface, and that is precisely what boundary integral techniques can do.

There are many ways boundary integral equations can be derived to determine the normal derivative of the velocity potential at the surface given the potential along it. A very successful method, and the one adopted in this paper, is presented in Baker et al. (1982) and used in many other studies of free surface flows, for example Baker et al. (1980), Baker (1983), Baker et al. (1987) and Baker \& Moore (1989). The basic idea in the method is to use a dipole distribution $\mu(p, t)$ along the surface to generate a complex velocity potential $\Phi=\phi+\mathrm{i} \psi$, where $\psi$ is the streamfunction, the conjugate harmonic function to $\phi$. In particular, for a water surface that is $2 \pi$-periodic, the complex velocity potential along the surface is given by

$$
\Phi(p)=\frac{1}{4 \pi \mathrm{i}} \int_{0}^{2 \pi} \mu(q) z_{q}(q) \cot \left(\frac{z(p)-z(q)}{2}\right) \mathrm{d} q+\frac{\mu(p)}{2},
$$

where the principal value of the integral must be taken. The Lagrangian parameter $p$ is chosen so that

$$
z(p+2 \pi)=p+z(p)
$$

a statement of the $2 \pi$-periodicity of the wave. Dependence on time has been suppressed in (2.1) and (2.2) for convenience.

The complex velocity $w=u+\mathrm{i} v$ at the surface determines its motion,

$$
\frac{\partial z}{\partial t}(p)=w(p)
$$

Partial time derivatives are used to emphasize that the motion is Lagrangian, that is, $p$ is kept fixed. The complex velocity along the surface is given by

$$
w^{*}(p)=\frac{\Phi_{p}(p)}{z_{p}(p)},
$$

where $w^{*}$ denotes the complex conjugate of the complex velocity $w$.

An evolution equation for the dipole strength is derived from the evaluation of Bernoulli's equation at the surface where the pressure is set to zero:

$$
\frac{\partial \mu}{\partial t}(p)=-2 \operatorname{Re}\left\{\frac{1}{4 \pi \mathrm{i}} f_{0}^{2 \pi} \frac{\partial \mu}{\partial t}(q) z_{p}(q) \cot \left(\frac{z(p)-z(q)}{2}\right) \mathrm{d} q\right\}+r(p),
$$

where

$$
\begin{aligned}
r(p)= & -2 \operatorname{Re}\left\{\frac{1}{4 \pi \mathrm{i}} f_{0}^{2 \pi} \mu(q) w_{p}(q) \cot \left(\frac{z(p)-z(q)}{2}\right) \mathrm{d} q\right\} \\
& +2 \operatorname{Re}\left\{\frac{1}{8 \pi \mathrm{i}} \int_{0}^{2 \pi} \frac{\mu(q) z_{p}(q)[w(p)-w(q)]}{\sin ^{2}(z(p)-z(q) / 2)} \mathrm{d} q\right\}+w^{*} w-2 g y .
\end{aligned}
$$

This is a Fredholm integral equation of the second kind for the rate of change of the dipole strength.

In summary, (2.3) and (2.5) constitute a set of evolution equations for $z$ and $\mu$. More details may be found in Baker et al. (1982). If $z$ and $\mu$ are known at some time $t,(2.1)$ 
may be evaluated to obtain the complex potential at the surface. Then the surface velocity $w$ may be obtained from (2.4) and the surface location updated by (2.3). Next, $r(p)$ may be evaluated and the integral equation for the rate of change of the dipole strength may be determined by (2.5).

Since the formulation of the boundary integral techniques involves a Lagrangian coordinate, even breaking surface waves can be tracked but only until the tip of the plunging wave reaches the surface below it. At this moment, the formulation fails. The surface re-connects through a complicated physical process.

Putting aside examples of topological changes, questions remain about whether the water surface can form curvature singularities in finite time. There are known examples of curvature singularities in water waves, for example the limiting form of the Stokes wave which has a corner of $2 \pi / 3$ radians at its peak and the limiting form for a standing wave that momentarily forms a corner of $\pi / 2$ radians. But these events appear to be very special cases. Can curvature singularities arise spontaneously under more general conditions? To shed light on this question, highly accurate numerical solutions to the evolution equations must be constructed with a view to studying the mathematical nature of the curvature.

\subsection{Numerical procedure}

Suppose that $z(p)$ and $\mu(p)$ are known at some time $t$ at equally spaced points in $p$. Denote as $z_{j}$ and $\mu_{j}$ the values at these points. There are four components to a numerical method that advances these values in time: numerical differentiation, evaluation of the surface integrals, solution of the integral equation and discrete time stepping. Each component will be described first before providing an outline of the numerical procedure.

Spectral representations present the most accurate numerical methods and since the motion of the water waves is assumed to be $2 \pi$-periodic, a Fourier series may be used for spectral accuracy. The functions $x(p)-p, y$ and $\mu$ are $2 \pi$-periodic functions of $p$ of the form

$$
f(p)=\hat{f}_{0}^{e}+\sum_{k=1}^{\infty}\left[\hat{f}_{k}^{e} \cos (k p)+\hat{f}_{k}^{o} \sin (k p)\right],
$$

where the superscripts $o, e$ denote the coefficients of the odd and even modes. Divide a $2 \pi$-period of $p$ into $N$ intervals of equal spacing $h=2 \pi / N$. A spectral approximation to $f(p)$ is the truncated sum evaluated at the discrete points $p_{j}=j h$ :

$$
f_{j}=\hat{f}_{0}^{e}+\hat{f}_{N / 2}^{e}(-1)^{j}+\sum_{k=1}^{N / 2-1}\left[\hat{f}_{k}^{e} \cos (k j h)+\hat{f}_{k}^{o} \sin (k j h)\right] .
$$

The fast Fourier transform (FFT) may be used to calculate the Fourier coefficients from the discrete function values, and its inverse transform restores the function values from the Fourier coefficients.

In several steps of the numerical procedure, derivatives must be calculated. Spectrally accurate derivatives may be obtained through the differentiation of the Fourier series (2.8). Denote the first and second derivative by $(\mathscr{D} f)_{j}=f_{p}(j h)$ and $\left(\mathscr{D}^{2} f\right)_{j}=f_{p p}(j h)$. Then

$$
(\mathscr{D} f)_{j}=\sum_{k=1}^{N / 2-1} k\left[\hat{f}_{k}^{o} \cos (k j h)-\hat{f}_{k}^{e} \sin (k j h)\right],
$$




$$
\left(\mathscr{D}^{2} f\right)_{j}=\sum_{k=1}^{N / 2-1} k^{2}\left[-\hat{f}_{k}^{e} \cos (k j h)-\hat{f}_{k}^{o} \sin (k j h)\right] .
$$

For analytic functions, the Fourier coefficients $\hat{f}_{k}^{e}$ and $\hat{f}_{k}^{o}$ decay exponentially in $k$. When calculated by the FFT, however, the tail of the spectrum $\hat{f}_{k}$ will settle at the level of round-off. When the spectral derivatives are sought numerically as in (2.9) and (2.10), the round-off errors will be multiplied by $k$, sometimes large depending on spatial resolution. This will lead to a serious misrepresentation of the Fourier spectrum for the derivative, especially in the tail of the spectrum where round-off errors usually dominate. If this process is repeated, as during the time evolution of the surface, then the tail of the spectrum can grow unstably, leading to an eventual failure of the calculations. To alleviate this problem it is essential to use smoothing techniques to manually control the growth of round-off errors in the tail of the spectrum. A standard approach is to replace (2.9) by

$$
(\mathscr{D} f)_{j}=\sum_{k=1}^{N / 2-1} k S(k)\left[\hat{f}_{k}^{o} \cos (k j h)-\hat{f}_{k}^{e} \sin (k j h)\right]
$$

to calculate the derivatives, where $S(k)$ is used to suppress the ill-posed effects due to round-off errors. In addition, aliasing errors may be introduced in the spectral calculation of derivatives without smoothing which can also lead to numerical instability (see Gottlieb \& Orszag 1977). Consequently, smoothing or filtering techniques have been used extensively.

In general, smoothing may be realized through a convolution with an appropriate function or by multiplying the Fourier coefficient with its Fourier transform $S$, for example see Krasny (1986). Krasny's choice of the function $S$ takes the form

$$
S\left(\left|\hat{f}_{k}\right|\right)= \begin{cases}1 & \text { if }\left|\hat{f}_{k}\right| \geqslant \epsilon_{0}, \\ 0 & \text { if }\left|\hat{f}_{k}\right|<\epsilon_{0},\end{cases}
$$

where $\epsilon_{0}$ is a prescribed level just above machine precision. A modified version of this sharp cutoff filter is built on the tanh function,

$$
S(\eta)=\frac{1}{2}\left[1+\tanh \left(\frac{\eta+L}{d}\right)\right],
$$

where $\eta=\log _{10}\left|\hat{f}_{k}\right|$, and $L$ and $d$ are location parameters controlling the position and width of the smooth transition in the tanh profile. Note that round-off errors in the Fourier coefficients will not be removed completely, but reduced to lower values. The filter (2.12) can be applied afterwards with values of $\epsilon_{0}$ just above the new round-off level to remove these amplitudes completely. The result has a smoother decay in the tail of the spectrum.

Alternatively, the function $S(k)$ in (2.11) is selected to be a fast decaying function of the wavenumber $k$, taking values $S(k)=1$ for small $k$ and $S(k)=0$ for large $k$. For example,

$$
S(k)=\frac{1}{2}\left[1-\tanh \left(\frac{2 k \pi / N-\xi_{0}}{d}\right)\right],
$$

where $1 \leqslant k \leqslant N / 2$ and $\xi_{0}$ locates the centre of the transition zone, usually as some fraction of $\pi$. The parameter $d$ controls the width of the transition zone. 
This filter is very efficient in reducing the high modes. However, poor choices of $\xi_{0}$ and $d$ may lead to unnecessary reduction of the amplitudes of lower modes that are still important. Another choice $S(k)=\exp \left[-10(k / N)^{25}\right]$ was introduced by Hou, Lowengrub \& Shelley (1994). More filtering options based on wavenumber are available in Vichnevetsky \& Bowles (1982).

In practice, the Fourier coefficients are calculated, multiplied by $S(k)$ and then transformed back to the physical domain. To calculate derivatives, just use (2.11) before the inverse transform. In the work reported here, all filters are tried, sometimes in combinations, and the parameters adjusted to ensure that a significant part of the spectrum is independent of filtering technique. Typically, the spectrum decays down to the filter level and there is no further part to the spectrum for higher wavenumbers. However, as resolution deteriorates, the tail of the spectrum can rise above the filter level and eventually the calculation will fail. The choice of parameters is delicate and it may require trial-and-error to pick the appropriate value so that a sufficient portion of modes are unchanged while the undesirable high modes are suppressed. Poor choices of parameters may lead to noticeable decreases in total energy per time step, which is a conserved quantity and is used as a diagnostic tool for checking the accuracy of the numerical results.

The integrals in (2.1) and (2.5) are principal-valued integrals. Numerical treatment of the integrals is vastly improved by removing the pole singularity in the integrand through an appropriate subtraction of a known principal-valued integral. To be specific, note that

$$
f_{0}^{2 \pi} z_{p}(q) \cot \left(\frac{z(p)-z(q)}{2}\right) \mathrm{d} q=0
$$

hence (2.1) may be rewritten as

$$
\Phi(p)=\frac{1}{4 \pi \mathrm{i}} \int_{0}^{2 \pi}[\mu(q)-\mu(p)] z_{p}(q) \cot \left(\frac{z(p)-z(q)}{2}\right) \mathrm{d} q+\frac{\mu(p)}{2} .
$$

The integrand in (2.16) is now analytic and thus amenable to the spectrally accurate composite trapezoidal rule. The appropriate limit of the integrand at the point $q=p$ must be used. One approach to bypass this requirement is the alternate point quadrature adopted in Baker (1983), where the trapezoidal rule skips over the point $q=p$, maintains spectral accuracy and reduces the operations by half. Specifically, the integral in (2.16) is approximated by

$$
\frac{1}{N \mathrm{i}} \sum_{k+j=\text { odd }}\left(\mu_{k}-\mu_{j}\right)(\mathscr{D} z)_{k} \cot \left(\frac{z_{j}-z_{k}}{2}\right),
$$

where $(\mathscr{D} z)_{k}=z_{p}(k h)$ uses the formula (2.11). All other integrals are handled in the same way, in particular the integrals in (2.6) that are used to solve for $\partial \mu / \partial t$. Strictly speaking, pole removal is not necessary for the alternate point quadrature, but it helps to keep round-off errors down since the integrand remains bounded. Otherwise, the points on either side of the pole singularity give large values of opposite sign in the integrand that may not be cancelled accurately enough.

Equation (2.5) must be solved for $\partial \mu / \partial t$. Fortunately, an efficient iterative method is available,

$$
{\frac{\partial \mu_{j}}{\partial t}}^{(n+1)}=-2 \operatorname{Re}\left\{\frac{1}{N \mathrm{i}} \sum_{k+j=\mathrm{odd}}\left({\frac{\partial \mu_{k}}{\partial t}}^{(n)}-{\frac{\partial \mu_{j}}{\partial t}}^{(n)}\right)(\mathscr{D} z)_{k} \cot \left(\frac{z_{j}-z_{k}}{2}\right)\right\}+r_{j},
$$


where the pole subtraction (2.15) and the alternate point quadrature are used. Provided the resolution of the surface is accurate, the method will converge and the rate of convergence is large when the surface is nearly flat. The numerical solution is considered found when the absolute difference between two successive iterates is smaller than a prescribed error tolerance $\epsilon_{1}$,

$$
\max _{j=1, \ldots, N}\left|\frac{\partial \mu_{j}^{(n+1)}}{\partial t}-\frac{\partial \mu_{j}^{(n)}}{\partial t}\right| \leqslant \epsilon_{1} .
$$

Generally, approximately 10 iterations are required so that the absolute difference between two successive iterates is smaller than $10^{-13}$. The cutoff filter level (2.12) is often set at this level. Convergence is improved when the initial iterate is picked as the solution from the previous time step.

After calculating the complex velocities $w$ and the time derivatives of the dipole strength $\partial \mu / \partial t$, the rates of change of the wave profile $z=x+\mathrm{i} y$ and the dipole strength $\mu$ are known and they may be updated by a suitable ODE solver. Many accurate and stable schemes are available. The standard fourth-order Runge-Kutta method is chosen in our algorithm.

The numerical method proceeds as follows. Knowing $z_{j}$ and $\mu_{j}$, the velocity potential at the surface (2.16) is evaluated by using (2.17) once the derivative $(\mathscr{D} z)_{j}$ has been determined from (2.11). Using (2.11) again determines $\Phi_{p}$ and hence the velocity (2.4). The time derivative of $z$ is now known (2.3). Next, (2.6) is evaluated and the integral equation solved by iteration (2.18). Thus the time derivative of $\mu$ is known and the time derivatives may be passed to the ODE solver.

The first-order Stokes wave, $2 \pi$-periodic and travelling to the right,

$$
\left.\begin{array}{l}
x(p)=p-\varepsilon \sin (p-\sqrt{g} t), \quad y(p)=\varepsilon \cos (p-\sqrt{g} t), \\
\mu(p)=2 \varepsilon \sqrt{g} \sin (p-\sqrt{g} t),
\end{array}\right\}
$$

provides a good test of the algorithm and the code. The time scale is set with $g=1$ and an initial condition is obtained by setting $t=0$.

Resolution studies confirm spectral accuracy in space and fourth-order accuracy in time. The expectation is that the numerical solutions converge to a solution of the full nonlinear equations and not the linear solution given above. The difference though should be $O\left(\varepsilon^{3}\right)$ according to higher-order terms in the Stokes expansion for a travelling wave of permanent form, and the numerical results confirm the expected behaviour.

It is difficult to verify completely the accuracy of the code for the fully nonlinear water wave problem simply because a general exact solution is unavailable. Fortunately there is an important invariant of the wave motion, which is the total energy. As in Baker et al. (1982), the kinetic energy $E_{K}$ and the potential energy $E_{P}$ may be expressed as

$$
\begin{aligned}
E_{K} & =\frac{1}{2} \int_{0}^{2 \pi} \psi \phi_{q} \mathrm{~d} q, \\
E_{P} & =\frac{g}{2} \int_{0}^{2 \pi} y^{2} x_{q} \mathrm{~d} q,
\end{aligned}
$$

where $\psi$ and $\phi$ are the streamfunction and potential defined in (2.1). The total energy per spatial period $E_{T}=E_{K}+E_{P}$ is conserved in time. The energy $E_{T}$ may 


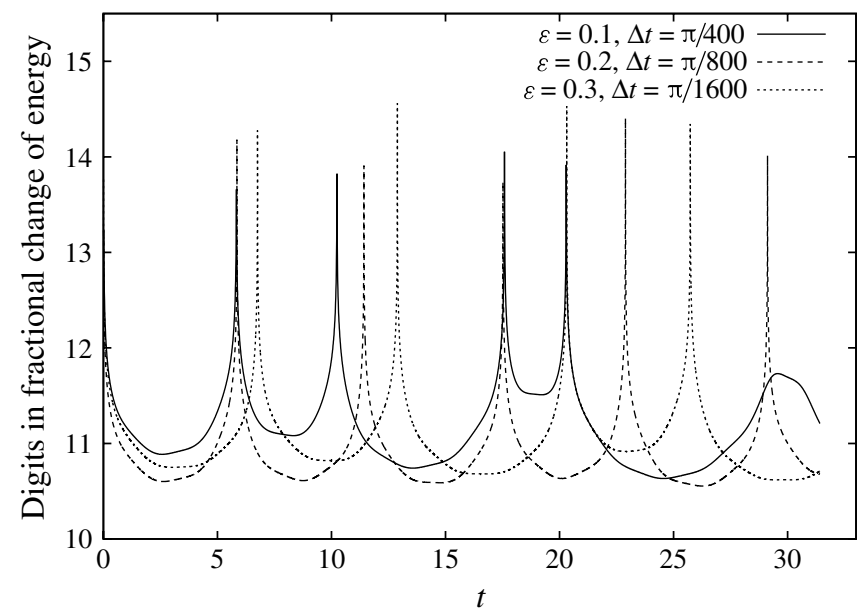

FIGURE 1. Number of digits in the fractional change of the total energy versus time, $\varepsilon=0.1,0.2,0.3$, different $\Delta t$.

be evaluated with spectral accuracy because it involves the numerical integration of periodic functions.

As a simple test, take (2.20) at $t=0$ with $\varepsilon=0.1,0.2,0.3$ as the initial conditions and execute the code with $N=64$ and $\Delta t=\pi / 400, \pi / 800, \pi / 1600$, respectively. Apply the tanh filter (2.13) with $L=12$ and $d=0.5$ and choose $\epsilon_{1}=10^{-13}$ in the convergence criterion (2.19). Run the code until $t=10 \pi$. Let $E_{T}(t)$ denote the total energy at time $t$. As in Ceniceros \& Hou (1998), monitor the number of digits in the fractional change of energy, that is,

$$
\text { number of digits }=-\log _{10} \frac{\left|E_{T}(t)-E_{T}(0)\right|}{\left|E_{T}(0)\right|} .
$$

Figure 1 shows the number of digits in the fractional change of the total energy versus time for the different cases. In all cases, the energy has about 11 digits of accuracy provided a small enough time step is used. Note that smaller time steps are needed for this level of accuracy as the amplitude is increased. The energy oscillates with a very small amplitude around the exact value causing the difference to be zero periodically, as revealed by the spikes in the graphs. We conclude that the method is very accurate and reliable.

\section{Travelling waves}

The first-order Stokes wave (2.20) provides an initial condition that is analytic in $p$ and is not already a breaking wave for all amplitudes $\varepsilon<1$. Of course, above the linear level, the wave does not retain its permanent form as nonlinear effects come into play during its motion. Indeed, as will be shown, these waves can break and form plunging tips of high curvature. The question, then, is whether curvature singularities can arise in finite time and if so, what is their nature?

To address these questions, a specific case of a plunging breaker will be studied in detail. Figure 2 shows the plunging breaker that forms for $\varepsilon=0.5$. The results are obtained through a series of calculations because the evolving surface requires increasing resolution for accuracy. The calculation starts with $N=2048$ 


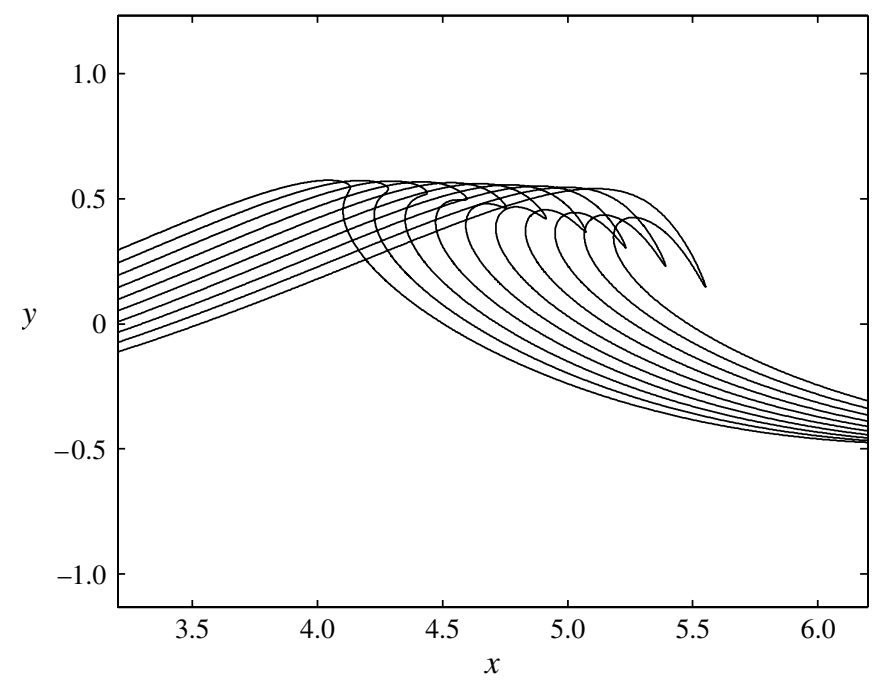

FIGURE 2. The plunging breaker when $\varepsilon=0.5$. The profiles are shown in sequence at $t=3.1,3.2, \ldots, 4.0$.

$\begin{array}{lc}\text { Interval } & N \\ 0 \leqslant t \leqslant 3.54 & 2048 \\ 3.54 \leqslant t \leqslant 3.8 & 4096 \\ 3.8 \leqslant t \leqslant 4.0 & 8192\end{array}$

TABLE 1. Spatial resolution in each time range.

and $\Delta t=0.0002$. The plain filter (2.12) with $\epsilon_{0}=10^{-11}$ is applied whenever a derivative is needed. The filter (2.14) is applied after each time step, with $\xi_{0}=\pi / 4$ and $d=\pi / 40$, to $x, y$ and $\mu$ to contain the growth of round-off errors. The tolerance level in (2.19) is set at $\epsilon_{1}=10^{-10}$. At $t=3.54$, the amplitudes of the Fourier spectrum begin to rise in absolute value above round-off errors near the end of the discrete Fourier range $N / 2=1024$. If the calculation is continued in time, accuracy is lost as the Fourier spectrum continues to rise in magnitude. Instead, the spatial resolution is doubled using interpolation based on the Fourier series. Now the spectrum is fully resolved but continues to rise in absolute value until another re-doubling is necessary. Table 1 presents the choice of $N$ during each time range. By increasing resolution, the number of digits of accuracy in the energy is better than 10 throughout the calculation. The time step remains at $\Delta t=0.0002$.

Quite clearly displayed in figure 2 is the increasing sharpness of the tip of the plunging breaker. The curvature of the surface is given by

$$
\kappa(p)=\frac{x_{p} y_{p p}-y_{p} x_{p p}}{\left(x_{p}^{2}+y_{p}^{2}\right)^{3 / 2}}
$$

and the curvature profile for the surface shown in figure 2 at the last time $t=4.0$ is displayed in figure 3 along with the time evolution of the minimum. The results 

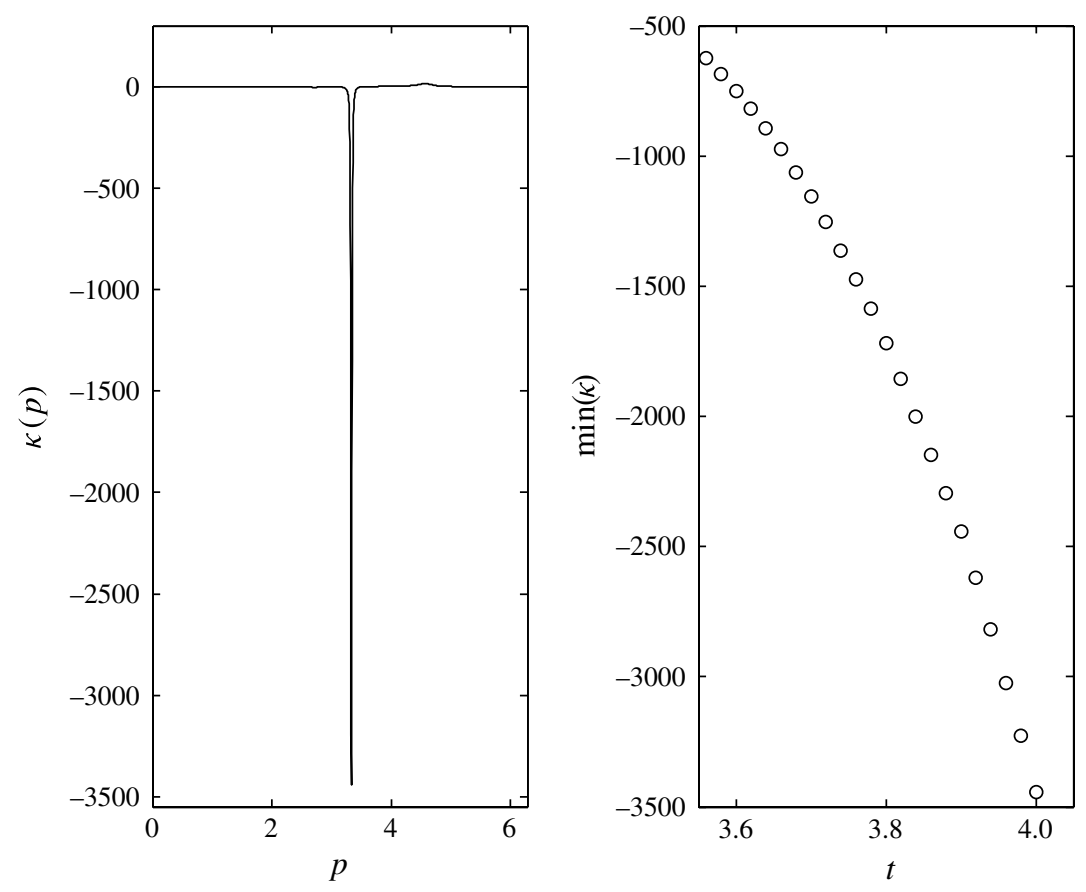

FIgURE 3. Profile of curvature at $t=4.0$ and time evolution of the minimum of curvature from $t=3.56$ to $t=4.0$ in increments of 0.02 .

show how narrow the tip is and how quickly it grows. This behaviour is certainly a candidate for the formation of a curvature singularity in finite time.

\subsection{Curvature singularities}

In general, it can be quite difficult to assess whether a singularity forms in finite time. One way forward that is both intellectually pleasing and practical is to search for isolated singularities in the complex $p$ plane. If found, they can be tracked to see whether they will reach the real axis in finite time. This approach has been used successfully in several studies of curvature singularity formation in free surface flows, for example, Krasny (1986), Shelley (1992), Baker et al. (1993), Nie \& Baker (1998), Ely \& Baker (1993) and Cowley et al. (1999). Basically, the method uses the asymptotic behaviour of the Fourier coefficients for large wavenumbers to detect the nearest isolated singularity in the complex plane; details are provided in Appendix A.

It is crucial that the Fourier spectrum is accurate over a wide range of wavenumbers to allow a successful form fit. Curvature spectra for the profiles in figure 2 are shown in figure 4 for $3.6 \leqslant t \leqslant 4.0$ with time increments of 0.1 . The regular pattern in the spectra confirms the possibility of a singularity in the complex physical plane because it has the appearance of the form (A 3). The sliding fit over $k$ is applied to the curvature spectrum at $t=3.6$ for example, and the results are shown in figure 5 . For a range in $k$, in particular $100<k<400$, the sliding form fit gives consistent results that approach limiting values. Estimates for the accuracy of the surface profile suggest the errors are about $10^{-11}$, which implies that the accuracy in the curvature is about $10^{-6}$. Even though the curvature spectra in figure 4 appear well resolved, the accuracy in the data limits the range where the form fit succeeds to $k<500$, the value where the curvature spectrum at $t=3.6$ reaches $10^{-6}$. The results displayed 


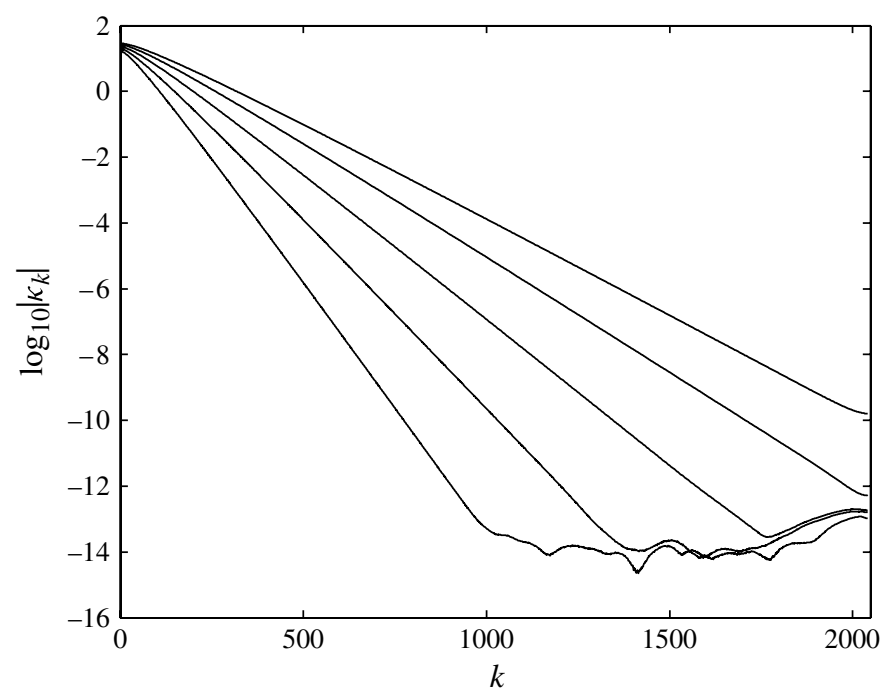

FIGURE 4. Curvature spectra expressed in the form $\left(\log _{10}\left|\widehat{\kappa}_{k}\right|\right)$. From bottom up the data are shown at $t=3.6,3.7,3.8,3.9$ and 4.0.

in figure 5 suggest that $\mu=-1.5, \delta=0.03585$, and then $\gamma=3.3161$ is calculated by the procedure described in Appendix A. The results were also checked with the least-squares fit and the results are consistent. Consequently,

$$
\kappa(p) \sim\left(p-p_{s}\right)^{-1.5},
$$

where $p_{s}=\gamma+\mathrm{i} \delta=3.3161+0.03585 \mathrm{i}$. As discussed in Appendix A, there is another singularity below the real $p$ axis which is the complex conjugate of the one above because the curvature is a real-valued function along the real $p$ axis. This will be true for all the results reported in this article, so for convenience only the singularity in the upper half-plane will be studied.

Having established the nature of the curvature singularity at $t=3.6$, the obvious next step is to verify its presence at all times and to determine if it reaches the real axis in finite time. So the sliding fit is applied to the curvature spectra at each time step. What is important in all the results is that irrespective of the time is, the exponent of the singularity $\mu$ is -1.5 . It is the location of the nearest singularity to the real axis, or the width of the analyticity strip, $\delta(t)$, that changes in time. Figure 6 shows the change in $\delta$, and compares its behaviour with the rapid decay of $1 / \max |\kappa|$. Initially, $\delta \approx 0.7$. As $t$ passes $0.5, \delta$ starts decreasing. As $t$ reaches 3 , the wave starts to break and the decay of $\delta$ slows down. As the tip of the plunging breaker becomes increasingly sharp, $\delta$ approaches the real axis but at an ever slowing pace and does not appear to reach the real axis in finite time, certainly not before the tip reaches the water surface below it.

One possible origin of the curvature singularity in the complex $p$ plane is that $x(p)$ and/or $y(p)$ have square-root singularities at $p_{s}$. However, attempts to fit the spectra of $x(p)-p$ and $y(p)$ fail to identify such a singularity. There must be another reason for the occurrence of curvature singularities. Since the curvature singularity is already present in the initial condition, it is worthwhile to explore the initial curvature. Note 

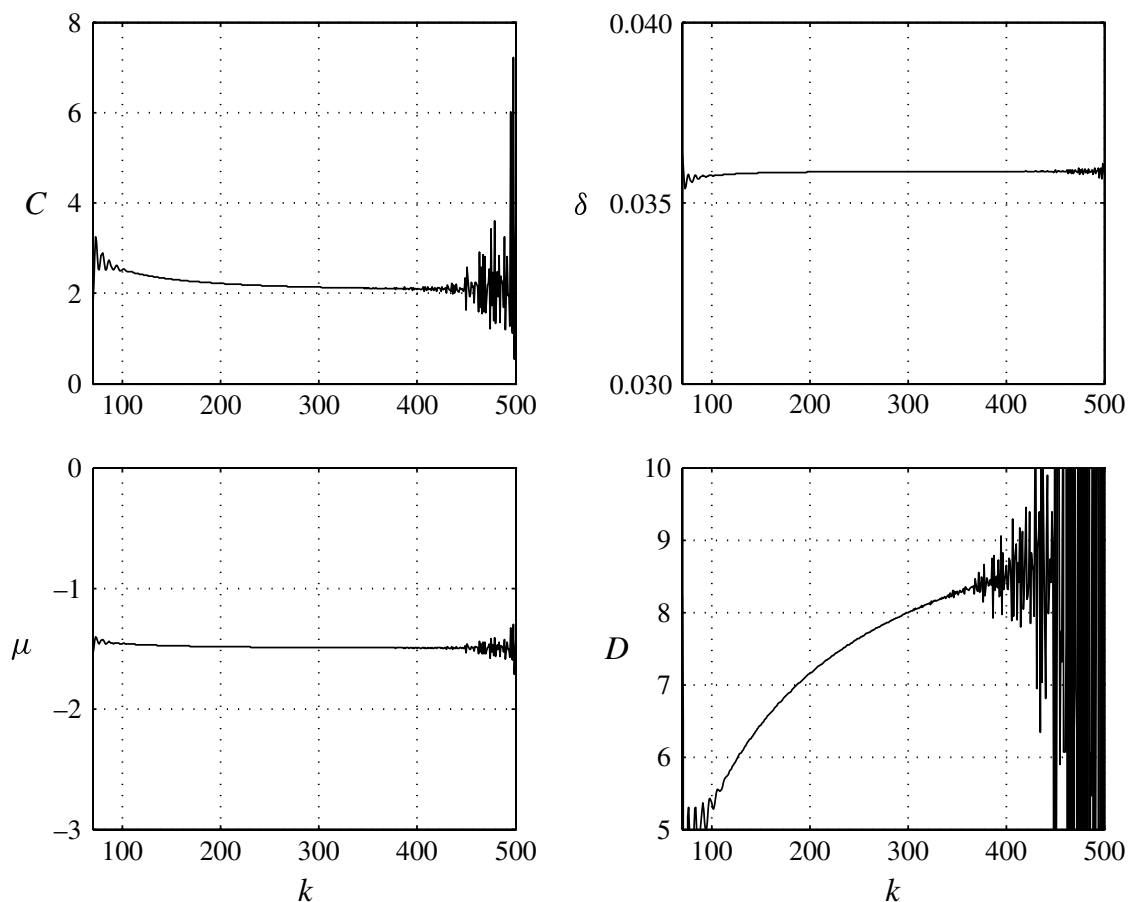

FIGURE 5. The parameters in the fit (A 3) to the spectrum of $\kappa(p)$ at $t=3.6$ for $\varepsilon=0.5$.
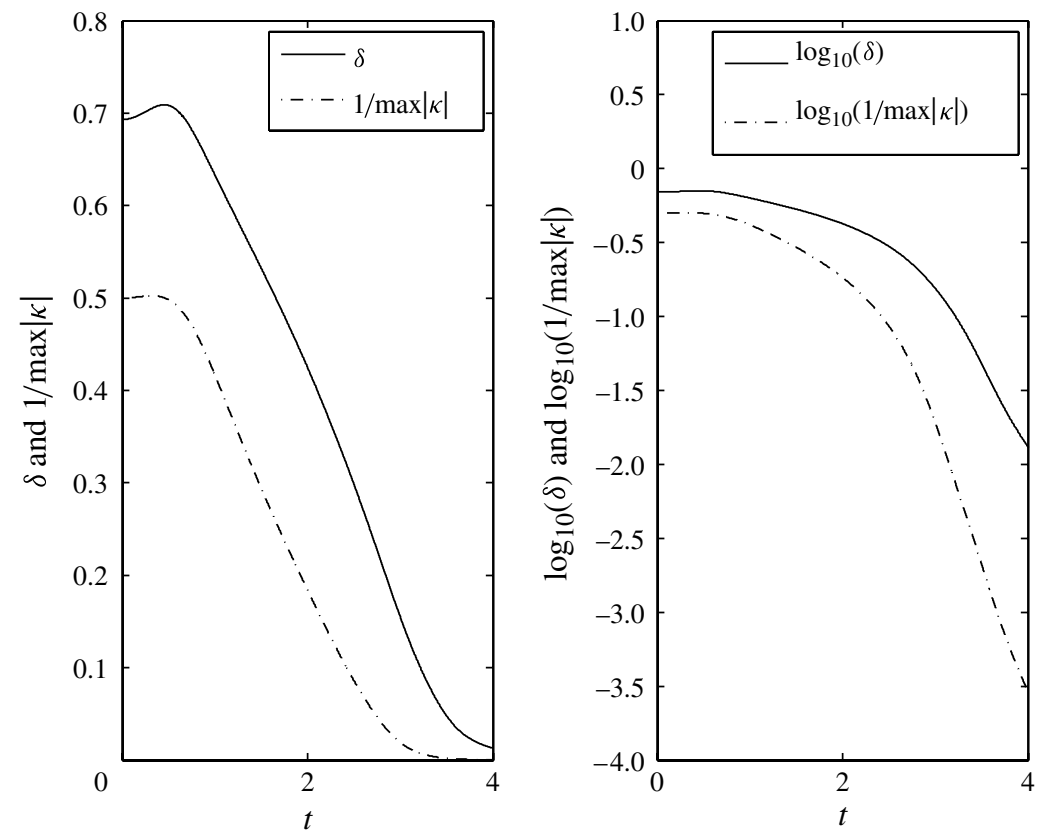

FIgURE 6. The quantities $1 / \max |\kappa|$ and $\delta$ as functions of $t$ for $\varepsilon=0.5$. 
first that the curvature (3.1) can also be expressed as

$$
\kappa=\frac{1}{s_{p}} \operatorname{Im}\left\{\frac{z_{p p}}{z_{p}}\right\}=-\frac{\mathrm{i}}{2 \sqrt{z_{p} \bar{z}_{p}}}\left(\frac{z_{p p}}{z_{p}}-\frac{\bar{z}_{p p}}{\bar{z}_{p}}\right) .
$$

In this form, it is easy to analytically continue $\kappa$ into the complex $p$ plane by replacing the complex conjugate $\bar{z}$ with $z^{*}(p)=\bar{z}(\bar{p})$ as done by Cowley et al. (1999). The initial condition (2.20) gives

$$
\begin{gathered}
z_{p}=1-\varepsilon \mathrm{e}^{\mathrm{i} p}, \quad z_{p p}=-\mathrm{i} \varepsilon \mathrm{e}^{\mathrm{i} p} \\
z_{p}^{*}=1-\varepsilon \mathrm{e}^{-\mathrm{i} p}, \quad z_{p p}^{*}=\mathrm{i} \varepsilon \mathrm{e}^{-\mathrm{i} p} .
\end{gathered}
$$

Clearly, $z_{p p}$ and $z_{p p}^{*}$ are analytic, but $z_{p}$ and $z_{p}^{*}$ have zeros, and these zeros lead to branch-point singularities in the curvature of power $-3 / 2$, exactly as found numerically. The zeros of $z_{p}$ and $z_{p}^{*}$ occur at $p=\mathrm{i} \ln (\varepsilon)$ and $p=-\mathrm{i} \ln (\varepsilon)$ respectively, which means it is the zero of $z_{p}^{*}$, in the upper half-plane that is detected numerically. For $\varepsilon=0.5$, the zero occurs at $0.693 \mathrm{i}$, which is the initial value shown in figure 6 .

The question naturally arises of whether this curvature singularity in the complex $p$ plane is simply an artifact of the parameterization. The matter is easily settled by considering the curvature as a function of the arclength variable $s$. For the initial condition (2.20), it is straightforward to determine the behaviour of the curvature in terms of the arclength. Consider the curvature near the point $p_{s}=-\mathrm{i} \ln (\varepsilon)$. First, note that

$$
\begin{aligned}
& z_{p} \sim 1+\varepsilon^{2}, \quad z_{p p} \sim-\varepsilon^{2} \mathrm{i}, \\
& z_{p}^{*} \sim \mathrm{i}\left(p-p_{s}\right), \quad z_{p p}^{*} \sim \mathrm{i},
\end{aligned}
$$

which means that

$$
s_{p}^{2}=z_{p} z_{p}^{*} \sim \mathrm{i}\left(1+\varepsilon^{2}\right)\left(p-p_{s}\right) \quad \text { or } \quad s-s_{0} \sim \frac{2}{3} \sqrt{\mathrm{i}} \sqrt{1+\varepsilon^{2}}\left(p-p_{s}\right)^{3 / 2},
$$

where the location of the singularity in the complex arclength plane is $s_{0}$. Since $z_{p p}^{*} / z_{p}^{*} \sim 1 /\left(p-p_{s}\right)$, the dominant contribution to the curvature near $p_{s}$ is

$$
\kappa \sim \frac{\mathrm{i}}{2 \sqrt{\mathrm{i}} \sqrt{1+\varepsilon^{2}}\left(p-p_{s}\right)^{3 / 2}} \sim \frac{\mathrm{i}}{3\left(s-s_{0}\right)} .
$$

The next step, then, is to determine the curvature as a function of the arclength and seek a form fit to its spectrum to locate any singularities and their nature. For this purpose it is necessary to redistribute the markers along the interface so that the new markers correspond to equal spacing in arclength. The procedure described in the Appendix of Baker \& Nachbin (1998) is followed. Once the new markers are located, the curvature values at these locations are found using Fourier interpolation. Take the data for $\varepsilon=0.5$ at $t=2.35$ as an example. The curvature spectra for the two distributions of markers are shown in figure 7 .

Clearly the spectra show a pattern that suggests that the form fit described in Appendix A is plausible. The issue is whether the data contain enough accuracy to produce consistent results. The results from the sliding fit procedure are shown in figure 8 . The power is clearly $\mu=-1$ and $C$ is near $1 / 3$, matching the predictions from (3.9). Recall that the data for the curvature are accurate enough when the spectrum reaches about $10^{-6}$ and from figure 7 this occurs near $k \approx 250$. Given this limitation, the form fit is certainly consistent. Indeed, it is usually easier to find consistent form fits when the curvature is expressed as a function of arclength. 


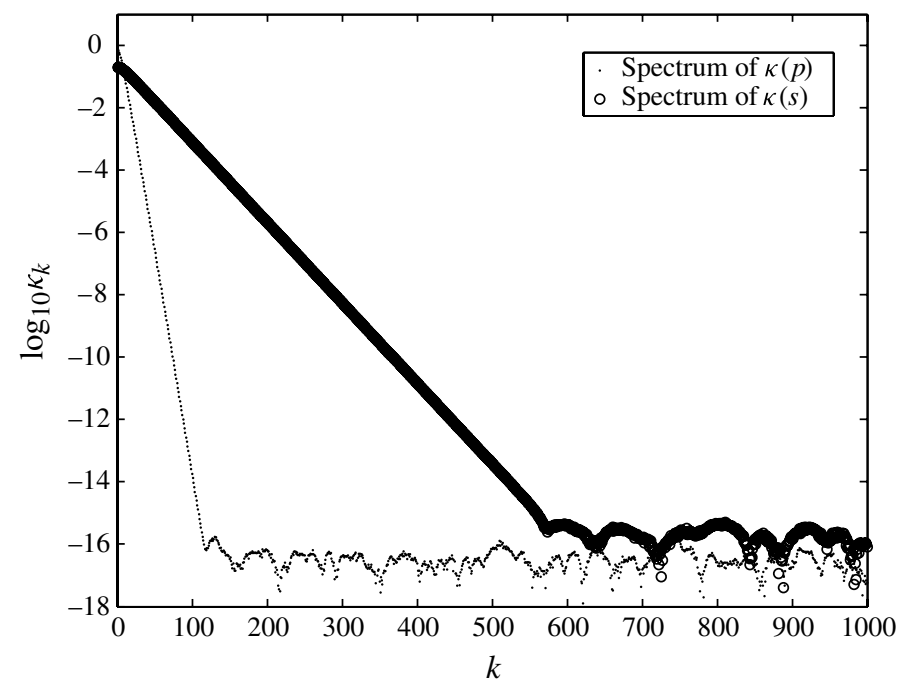

FIGURE 7. Curvature spectrum at $t=2.35$ for $\varepsilon=0.5$ with two sets of markers.
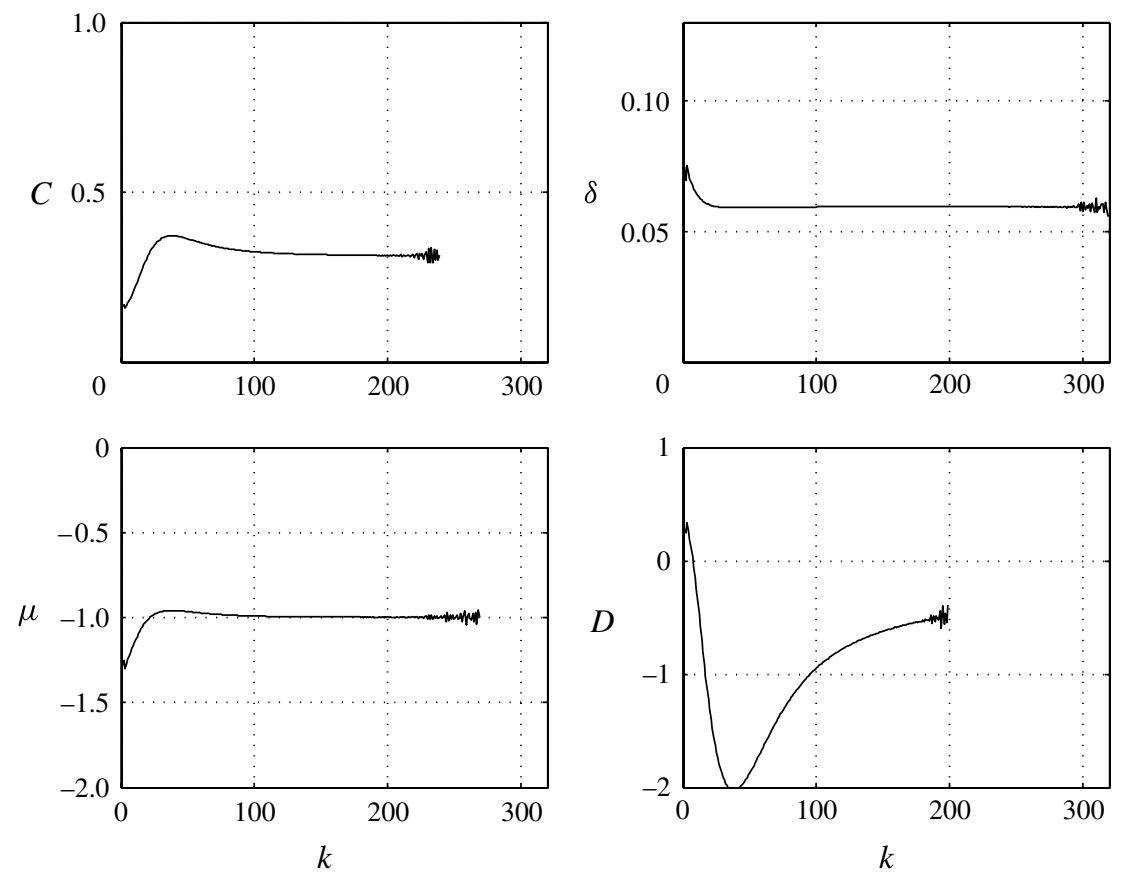

FIGURE 8. The parameters in the fit (A 3) to the spectrum of $\kappa(s)$ at $t=2.35$ for $\varepsilon=0.5$.

The sliding fit is applied to the calculated curvature spectra for each time at which reliable data are available, and the results give the power as $\mu=-1$ in all cases. The distance $\delta$ of the pole singularity from the real axis is shown in figure 9. Compared to figure 6 , the pole singularity is nearer to the real axis in the complex arclength plane than the 3/2-power singularity in the complex $p$ plane. Indeed, it becomes extremely difficult to fit the data at later times because the singularity is so much closer. The 

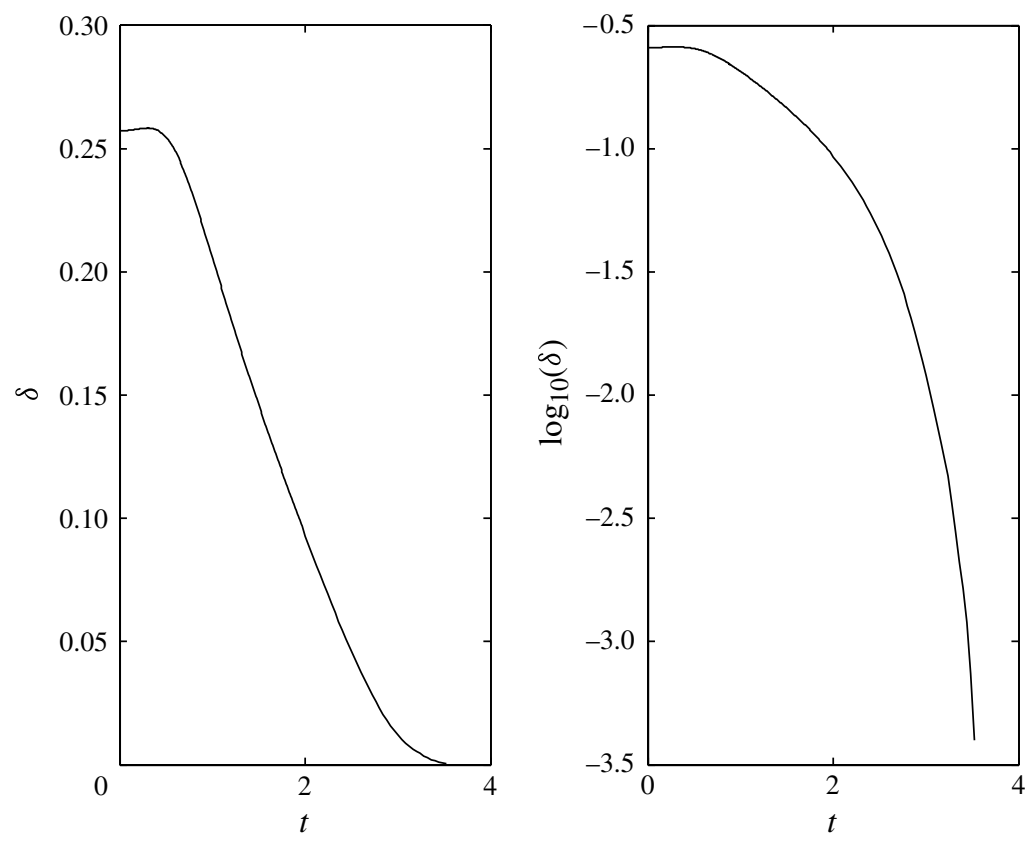

FIGURE 9. The quantities $\delta$ and $\log _{10}(\delta)$ as functions of $t$ for $\varepsilon=0.5$.

decrease of $\delta$ appears slightly faster than exponential but given that the singularity in the complex $p$ plane is slower than exponential, the evidence on whether a singularity forms in finite time is inconclusive. Presumably, the tip of the plunger where the curvature singularity might form is falling freely under gravity. The evidence in Baker et al. (1993) and Tanveer (1993) suggests no curvature singularity formation in finite time for a falling spike undergoing Rayleigh-Taylor instability. Certainly, a more definite study of falling spikes would be desirable to settle the matter, but it may be only of mathematical interest since surface tension effects will come into play long before singularity formation.

The evidence for pole singularities in the complex arclength plane is not just numerical. The theoretical work of Tanveer (1993) also predicts these singularities as generic in nature. In his work, Tanveer uses a conformal map from the unit disk into a periodic strip below the water surface in the physical plane. With $\eta$ the complex variable inside the unit disk, the mapping takes the form

$$
z(\eta, t)=2 \pi+\mathrm{i} \ln (\eta)+\mathrm{i} f(\eta, t),
$$

where $f(\eta, t)$ is analytic in a region that contains the unit disk and may have squareroot singularities outside the unit disk. In particular, the general expansion near a generic singularity is

$$
f(\eta, t)=\sum_{n=0}^{\infty} d_{n}(t)\left(\eta-\eta_{s}\right)^{n / 2} .
$$

Thus,

$$
z_{\eta} \sim \frac{\mathrm{i} d_{1}}{2 \sqrt{\eta-\eta_{s}}}, \quad z_{\eta}^{*} \sim b_{1},
$$




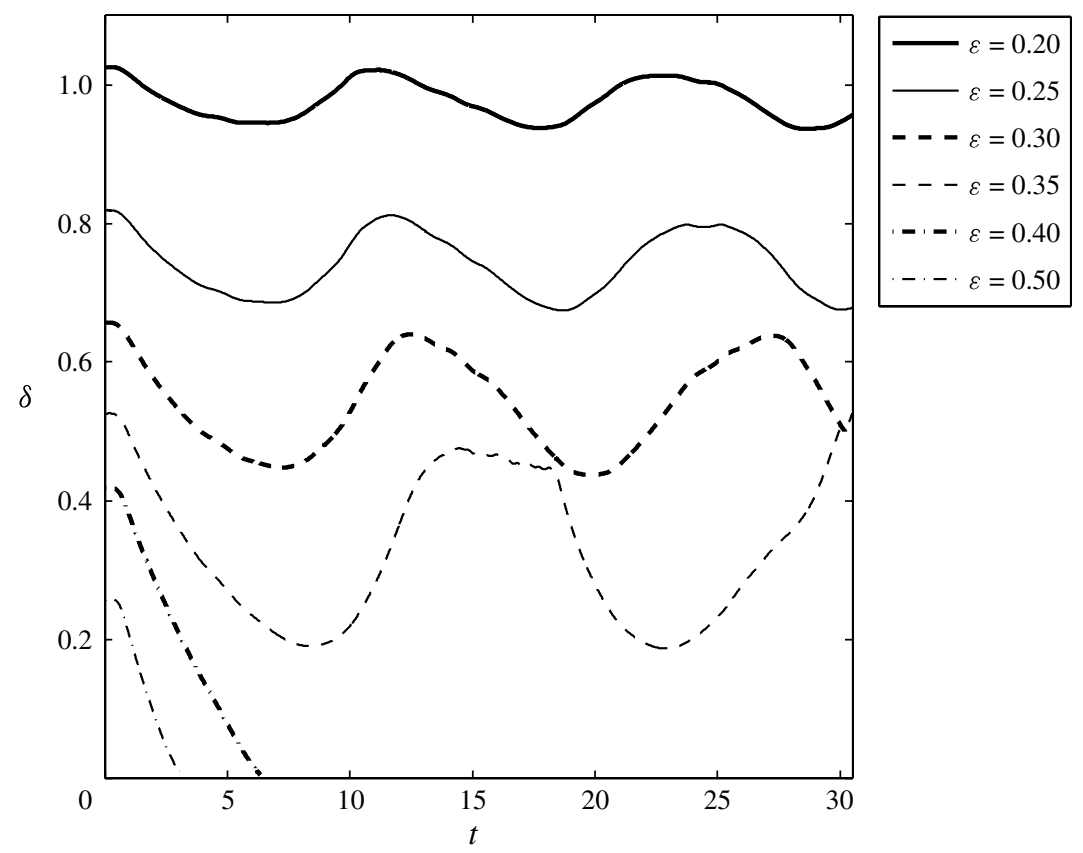

FIGURE 10. The distance $\delta(t)$ from the real axis of the curvature singularity in the complex $s$ plane for different $\varepsilon$.

where $z^{*}=\bar{z}(1 / \eta)$ is the analytic continuation of complex conjugation and it has no singularity at $\eta_{s}$. Furthermore,

$$
z_{\eta} z_{\eta}^{*}=s_{\eta}^{2} \sim \frac{\mathrm{i} b_{1} d_{1}}{2 \sqrt{\eta-\eta_{s}}}, \quad s-s_{0} \sim \frac{4 \sqrt{\mathrm{i} b_{1} d_{1}}}{3 \sqrt{2}}\left(\eta-\eta_{s}\right)^{3 / 4},
$$

which means that the analytically continued curvature (see (3.3)) is dominated by

$$
\kappa=-\frac{\mathrm{i}}{2 \sqrt{z_{\eta} z_{\eta}^{*}}}\left(\frac{z_{\eta \eta}}{z_{\eta}}-\frac{z_{\eta \eta}^{*}}{z_{\eta}^{*}}\right) \sim \frac{\mathrm{i}}{2 \sqrt{2 \mathrm{i} b_{1} d_{1}}}\left(\eta-\eta_{s}\right)^{-3 / 4} \sim \frac{\mathrm{i}}{3\left(s-s_{0}\right)},
$$

a result that agrees with (3.9). In summary, note that the square-root singularity in $z(\eta)$ corresponds to a zero in $z_{p}^{*}$ and both lead to a pole singularity in $\kappa(s)$. As an additional check, the conformal map between $\eta$ and $p$ is constructed numerically for a few profiles and it is verified that a square-root singularity in $z(\eta)$ maps to a zero in $z_{p}^{*}(p)$.

So far, results have been shown for the case $\varepsilon=0.5$. Numerical simulations and analysis of the results are also conducted for a range of $\varepsilon$. In all cases, the detection of singularities gave the same type of singularities in the complex $p$ and arclength planes. The main difference is in the trajectory of these singularities. In particular the distance $\delta$ of the curvature pole singularities from the real axis in the complex arclength plane are shown in figure 10 for various amplitudes $\varepsilon$. For small enough amplitudes, the waves do not break, and the pole singularity remains bounded away from the real axis. Periodically the waves develop smooth crests, observable when the pole singularity approaches the closest to the real axis. For $\varepsilon=0.4,0.5$, the waves break, and the pole singularity approaches the real axis rapidly. 


\subsection{Singularities in local models}

In addition, local solutions have been found that match the surface profile at the tip (Longuet-Higgins 1980) and the curling forward face of a breaking wave (LonguetHiggins 1982). In Appendix B, we reproduce the solution found by Longuet-Higgins (1980) but in terms of Lagrangian motion. We show that

$$
z(p, t)=[a \sinh (p+f)+\mathrm{i} b \cosh (p+f)] \mathrm{e}^{\mathrm{i} \delta}-\frac{g t^{2}}{2}
$$

is an exact solution to Euler's equation with free surface boundary conditions provided that the real-valued functions $a(t), b(t), f(t)$ and $\delta(t)$ satisfy certain first-order differential equations. Of course, the Lagrangian surface variable $p$ will be different from the one introduced by the initial condition (2.20), but since $z(p, t)$ in (3.15) is analytic in $p$, it is only where $z_{p}$ or $z_{p}^{*}$ have zeros that there are curvature singularities and they will be of the same type as in (3.9). Specifically, $z_{p}$ has a zero at

$$
p=-f+\mathrm{i} \epsilon, \quad \tan (\epsilon)=\frac{a}{b} .
$$

The zero lies directly above the location of the maximum curvature on the real $p$-axis. Unfortunately, since the definitions of $p$ in (3.15) and in the results displayed in figure 6 are different, a detailed comparison is not possible. Nevertheless, the trend in $\delta$ is similiar to that predicted by (B 18).

The location in the complex arclength plane can be determine from the integral of $s_{p}$,

$$
s_{0}=\sqrt{2} \rho \int_{-f}^{-f+\mathrm{i} \epsilon}[\cosh (2(p+f))-\cos (2 \psi)]^{1 / 2} \mathrm{~d} p,
$$

where the origin of the arclength is picked to coincide with the maximum in the curvature at $p=-f$. For small $\epsilon$,

$$
s_{0}=\mathrm{i} \frac{\pi}{2} \rho \epsilon^{2} \approx \frac{C}{\left(C_{1} t+1\right)^{3}},
$$

where we use the approximations (B 18). Given the limited range in time $3.5<t<4.0$ for which this approximation may be valid, a detailed match with numerical results is not possible. Nevertheless, it does account for the close approach to the real axis without the pole singularity reaching it in finite time.

A different local model developed by Longuet-Higgins (1982) captures the shape of the forward curling part of a breaking wave very well. The derivation uses the fact that

$$
\frac{\partial^{2} z}{\partial t^{2}}+\mathrm{i} g=\mathrm{i} R(p, t) \frac{z_{p}}{s_{p}} .
$$

Recall that the partial derivative in time keeps $p$ fixed; in other words, the motion is Lagrangian. The statement (3.19) has a direct connection to the Taylor condition since

$$
R=\frac{x_{p}}{s_{p}}\left(\frac{\partial^{2} y}{\partial t^{2}}+g\right)-\frac{y_{p}}{s_{p}} \frac{\partial^{2} x}{\partial t^{2}}=\boldsymbol{n} \cdot\left(\boldsymbol{x}_{t t}-\boldsymbol{g}\right)
$$

measures the normal component of the upper acceleration of a fluid particle on the surface which must be less than the gravitational acceleration. In other words, for the normal pointing away from the fluid, $R>0$. In figure 11, the profile of $R(p)$ for the 


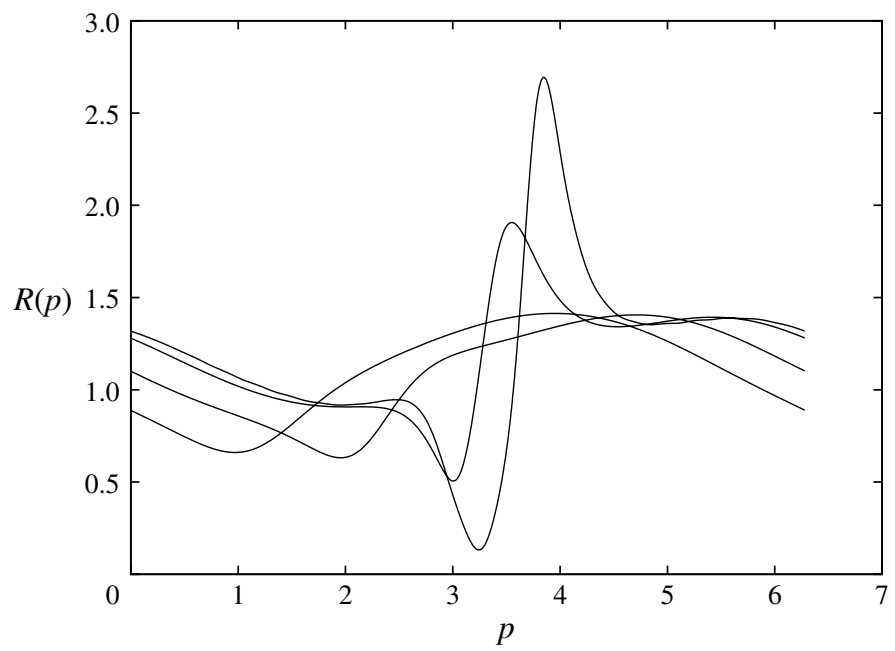

FIgURE 11. Profiles of $R(p)$ for the travelling wave with $\varepsilon=0.5$ for a sequence of times: $t=1,2,3,3.3$. The profiles shift to the right with the travelling wave.

wave shown in figure $2(\varepsilon=0.5)$ is shown for a sequence of times $t=1,2,3,3.3$. In all cases $R(p)>0$, even when the wave has clearly broken at $t=3.3$ (as shown in figure 2). However, $R(p)$ approaches zero at the tip of the plunging breaker, located near $p=3.2$, indicating that the tip approaches free fall, one of the assumptions in Longuet-Higgins (1980).

In Longuet-Higgins (1982), the assumption is made that $r(p, t)=R(p, t) / s_{p}(p, t)$ is only a function of time. Since $s_{p}$ is more or less uniform, but small between $p=3.2$ and $p=4.2$, the region of the forward face, $r$ follows $R$ closely except that its values are much larger. It looks like an inverted quadratic peaking near $r=7$. On the other hand, even if $r$ is a quadratic, it is possible that $z$ is a cubic as assumed in Longuet-Higgins (1982). Specifically,

$$
z=\frac{1}{3}-3 p^{2}+\mathrm{i}\left(2 p-p^{3}\right) .
$$

Longuet-Higgins shows that this cubic, tilted appropriately, matches experimental observation of the surface of a plunging breaker very well, a plunging breaker very similar to the one shown in figure 2.

The main interest here is the nature of the singularity in the approximation (3.21). Clearly $z(p)$ is analytic but $z_{p}$ has zeros at $\mathrm{i}(1 \pm 1 / \sqrt{3})$. The one closest to the real axis induces a pole singularity in the complex arclength plane at $s_{0}=0.66 \mathrm{i}$. This pole singularity is much further from the real axis than the one that is associated with the high curvature at the tip of the plunging breaker. Nevertheless, it is now clear that multiple pole singularities are possible in water wave propagation. In the next section, direct evidence for multiple poles will be presented.

\subsection{Profile singularities}

It might seem that the rapid approach to the real axis of pole singularities in the curvature is a signal that breaking waves have occurred. But it also seems plausible that pole singularities might approach the real axis as a signal that waves are cresting. This topic is reserved for the next section. Instead, consider breaking as the occurrence of the wave slope becoming vertical and the wave profile becoming multivalued as 

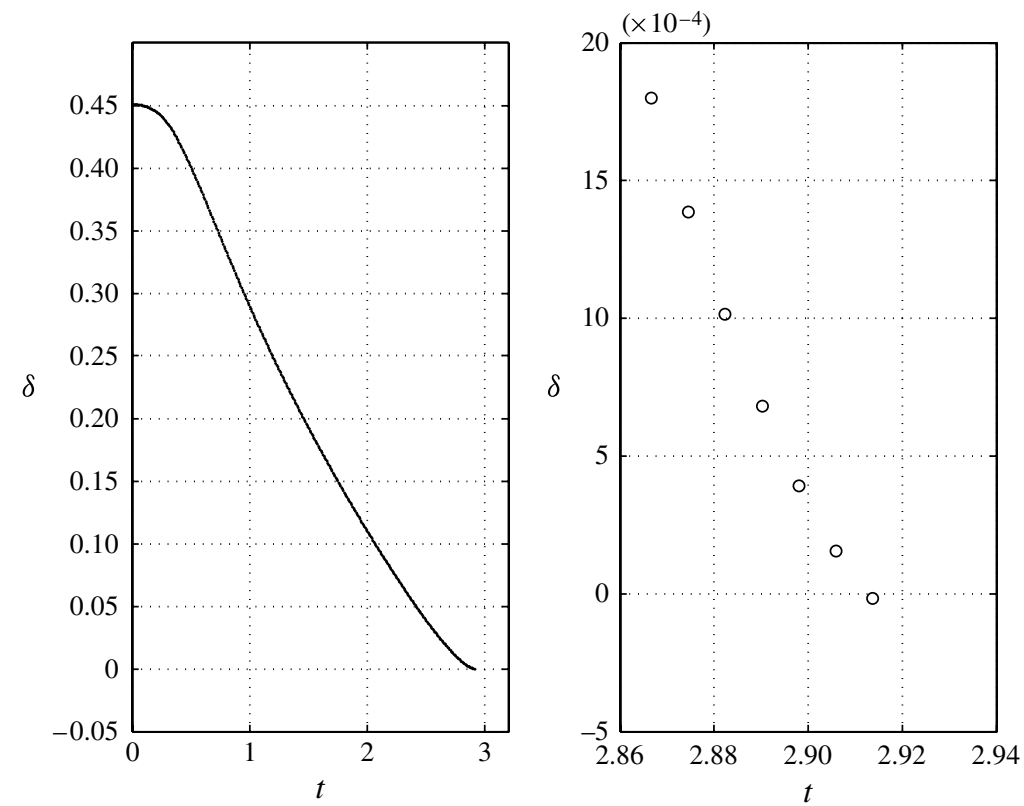

FIGURE 12. Vertical distance to the real axis of the profile singularity as a function of $t$ for $\varepsilon=0.5$, and an enlarged view.

the wave folds over. It is then appropriate to ask whether $y_{x}(x, t)=y_{p}(p, t) / x_{p}(p, t)$ becomes infinite in finite time. Indeed, we can ask a more general question. Are there any $y(x)$ singularities in the complex $x$ plane? We can answer this question by seeking a form fit to the Fourier coefficients of $y(x)$ given by

$$
y_{k}=\frac{1}{\pi} \int_{0}^{2 \pi} y(p) \mathrm{e}^{\mathrm{i} k x(p)} x_{p}(p) \mathrm{d} p .
$$

A sliding fit of the spectrum $\widehat{y}_{k}$ matches very well to

$$
y_{k}(t) \sim C(t) \mathrm{e}^{-\delta(t) k} k^{-1.5},
$$

indicating that $y$ has the following asymptotic expansion near $x_{s}$ :

$$
y(x) \sim \sqrt{x-x_{s}},
$$

where $x_{s}=\gamma(t)+\mathrm{i} \delta(t)$ is the singularity location in the complex $x$ plane. Since this singularity appears in $y$ as a function of $x$, it may be called a 'profile singularity'.

The distance $\delta$ of the square-root singularity from the real $x$ axis is shown in figure 12 for the case $\varepsilon=0.5$, a case where the wave breaks. Clearly, the singularity moves towards and reaches the real axis at about $t=2.91$. At this moment, the wave becomes vertical and thereafter the wave folds over. The role of the profile singularity and the curvature singularity becomes more transparent when their distances from the real axis are compared as in figure 13. Even though the distances are measured in different complex planes, they both clearly approach the respective real axes prior to the wave breaking. Once the wave breaks the curvature singularity slows down noticeably as a plunging tip is formed and falls freely under gravity. 


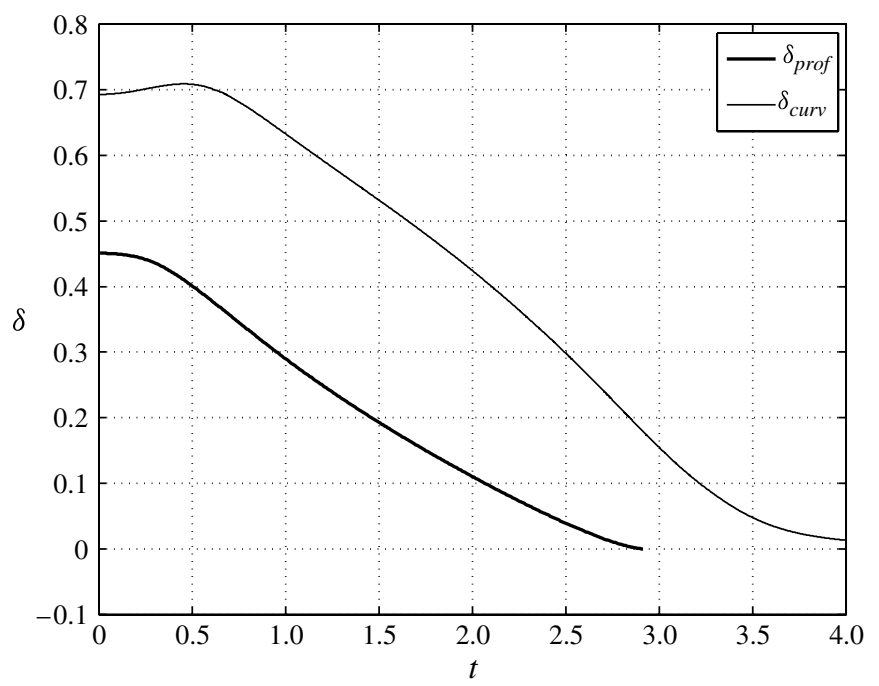

FIGURE 13 . The distances $\delta(t)$ of the profile singularity and the curvature singularity from their respective real axes for $\epsilon=0.5$.

Square-root singularities in the profile are found for all the choices of $\varepsilon$ that were made. Their distances from the real axis of the $x$-plane are shown in figure 14. The pattern is clear. When the waves break, the profile singularity reaches the real axis in finite time. When they do not, the profile singularity stays away from the real axis. But there is a different feature in the results. When the profile singularity for non-breaking waves recedes periodically, the wave flattens out somewhat. The form fit is much less reliable and the evidence suggests the possibility of more singularities appearing at comparable distances from the real axis. The form fit is designed to identify only the nearest singularity to the real axis. A different form is needed when there are more singularities; see Cowley et al. (1999). If this is true, then a simple connection of a single square-root singularity with a single travelling wave is not possible. On the other hand the presence of multiple square-root singularities offers a different view of the origin of the wave spectrum. Each singularity induces a tail in the spectrum of the form (A 4) and the wave spectrum is the result of their accumulated effects. Since the singularities capture the fully nonlinear effects of wave motion, even the exponentially small effects, the resulting spectrum can be significantly different from that generated by weakly nonlinear theories.

In order to remove the possibility that the presence of these singularities depends on the choice of initial condition (2.20), other initial conditions are used, in particular, $x(p)=p$ replaces the choice in (2.20). The same singularities are found and their behaviour is similar. We also used the initial condition in Fontelos \& de la Hoz (2010), and we find a pole singularity in the curvature in the complex arclength plane.

For travelling waves that break, a pole singularity in the curvature approaches the real axis very closely. The question arises of whether these singularities can approach the real axis under different circumstances. Two candidates immediately spring to mind: the corner singularity that occurs in the limiting form of the Stokes wave and the momentary formation of a corner singularity for the limiting form of standing waves. We have not succeeded in finding the instantaneous formation of corner singularities in travelling waves, although we have not conducted an exhaustive search. 


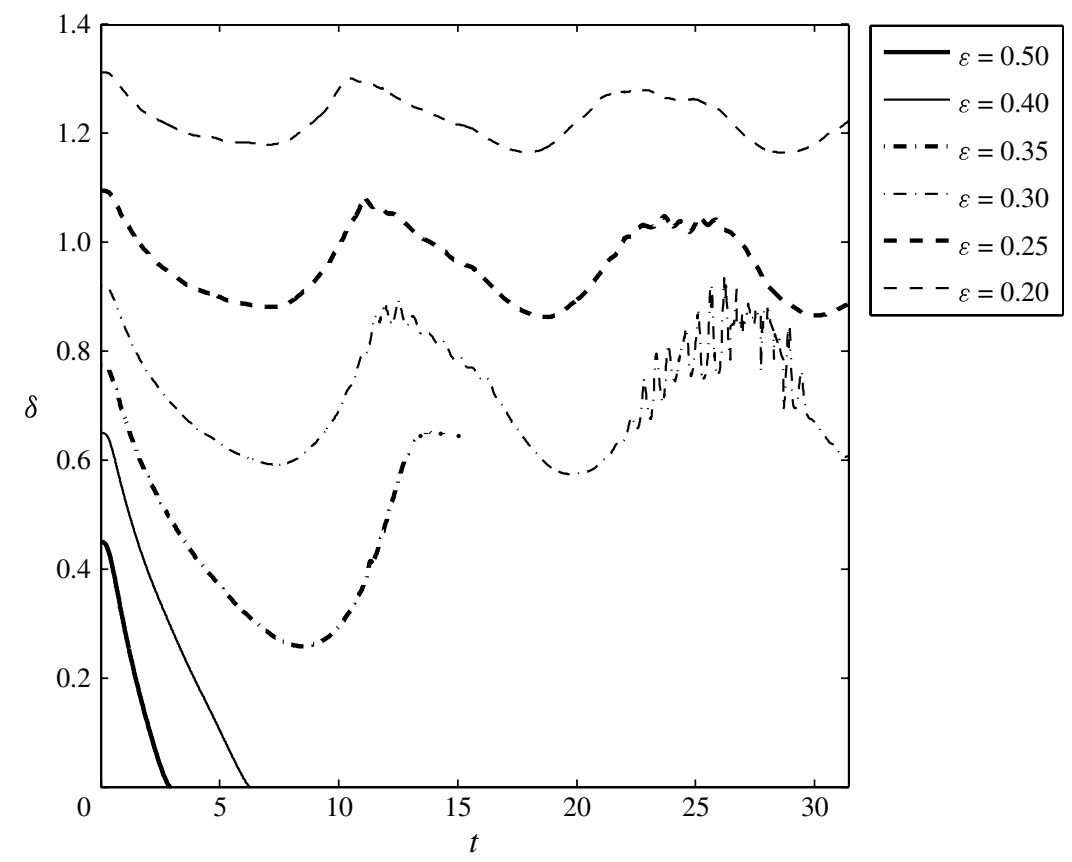

FIGURE 14. The distance $\delta(t)$ of the profile singularity from the real $x$ axis for different $\varepsilon$.

Alternatively, large-amplitude standing waves may well develop corner singularities and we explore this possibility in the next section.

\section{Standing waves}

Standing waves are waves that do not propagate. The simplest example is the wave trapped between two vertical boundaries. The linear wave,

$$
x(p)=p, \quad y(p)=\varepsilon \cos (p) \cos (t), \quad \phi(p)=-\varepsilon \exp (y) \cos (p) \sin (t),
$$

is a standard example of a standing wave. Note that the wave profile is even and $2 \pi$-periodic. One can easily imagine vertical boundaries at $x=0$ and $x=2 \pi$, but for our purposes it is better to regard the wave profile as periodic with certain symmetries. After one period, the wave returns to exactly the same shape, and it is possible to calculate waves of larger amplitude with this property. For example, Penny \& Price (1952) calculated a perturbation expansion to fifth-order using the wave amplitude as the parameter. Mercer \& Roberts (1992) use a numerical method to calculate two-dimensional standing water waves of larger amplitudes. In this section, we are interested in whether corner singularities can arise spontaneously starting with the initial condition in (4.1) but with large amplitudes.

For low amplitudes, the waves show a gradual adjustment due to nonlinear effects. Far more interesting is the case $\varepsilon=0.5$. Figure 15 shows successive profiles of the surface location. Observe that the profiles are plotted at approximately one-eighthperiod increments so it is easy to see the wave motion. The resolution is set as $N=1024, \Delta t=\pi / 4000$. The tanh filter (2.13) is applied with $L=12$ and $d=0.5$ whenever a derivative is calculated and in the smoothing procedure after each time step. The error tolerance $\epsilon_{1}=10^{-13}$ is used in the iterative solution for $\partial \mu / \partial t$. The 

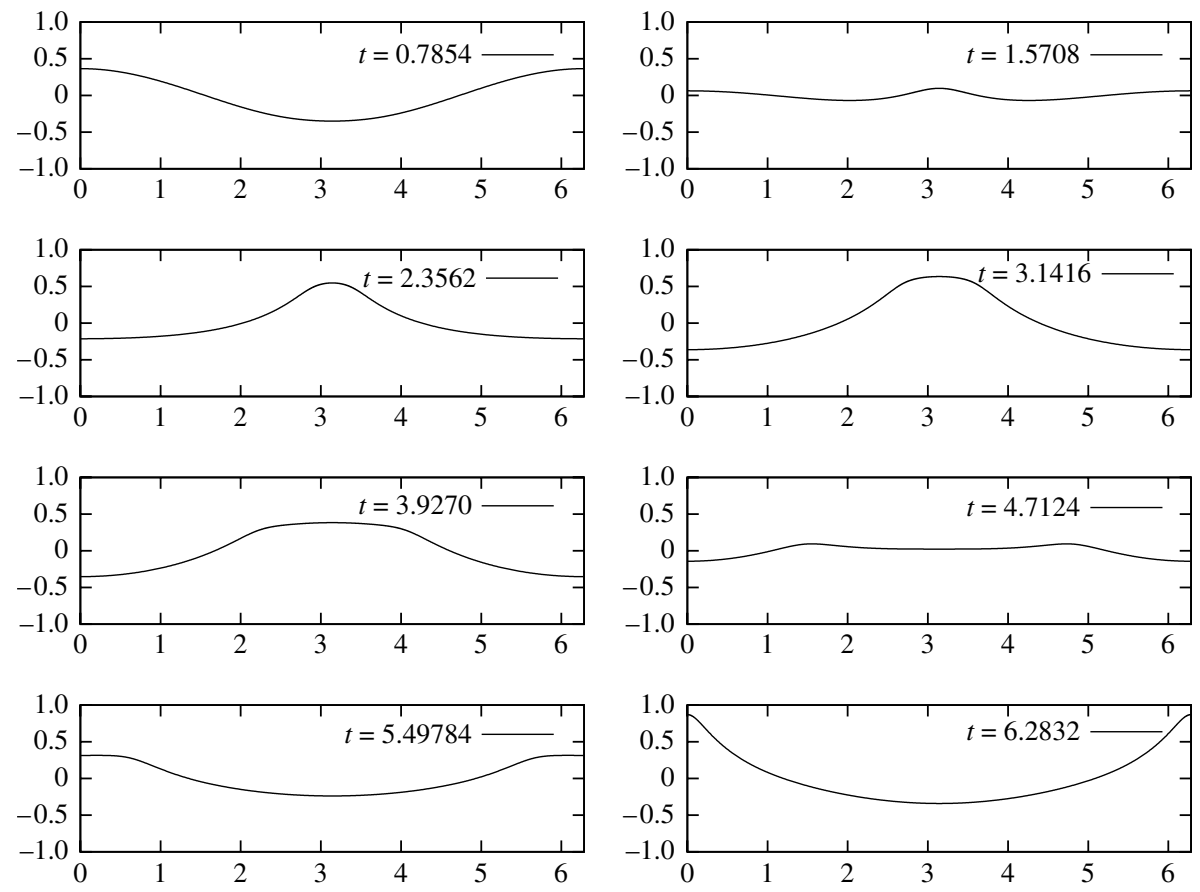

FIGURE 15. Successive wave profile plots for $\varepsilon=0.5$.

total energy $E_{T}(t)$ remains conserved to more than 10 digits. After about a half-period, a new crest has emerged at $x=\pi$, which subsequently splits into two outward moving waves. These waves collide at $x=0,2 \pi$ and form high peaks at the two ends. After reaching a maximum height the peaks begin to collapse rapidly.

More resolution is needed to continue the calculations with high accuracy. A restart is performed at $t=7.854$ with $N=2048$ and $\Delta t=\pi / 20000$. The collapsing peak at $x=0$ is shown in detail in figure 16 at successive times from $t=2.175 \pi$ to $t=2.75 \pi$. As the peak collapses it sharpens with high curvature developing at the peak, but eventually the peak is absorbed back into the background wave profile and disappears. Similar patterns in behaviour have been calculated before by Longuet-Higgins \& Dommermuth (2001).

The dynamics of the process is revealed by considering the trajectory of the Lagrangian marker centred on the peak at $x=0$. The height of the marker, its vertical velocity and acceleration are shown in figure 17. During the collapsing phase, from about $t=2 \pi=6.28$ until $t=2.7 \pi=8.48$, the particle acceleration is very close to $g=-1$, which means the peak is falling almost freely under gravity. Once the peak is absorbed in the background wave profile, the Lagrangian particle at the crest location suffers a rapid deceleration, driven presumably by a pressure gradient that overcomes the downward falling motion. The upward acceleration is much greater than gravity.

Prior to examining the role that curvature singularities in the complex plane may have, it is instructive to observe the curvature profiles in time. In figure 18 the curvature profiles are shown for the time period covering one oscillation of the standing wave, from an early time $t=0.75 \pi=2.36$ until $t=2 \pi=6.28$, a time just before the start of the collapse of the peak. Already by $t=0.75 \pi=2.36$, the curvature shows two spikes, placed evenly about $x=0(p=0)$. These spikes move towards 


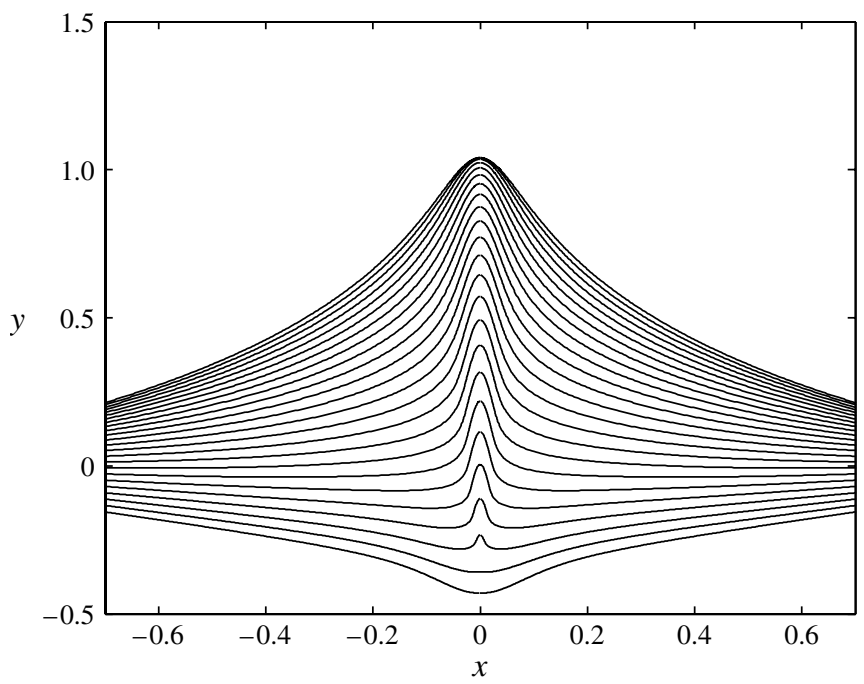

FIGURE 16. Detailed of the collapsing peak from $t=2.175 \pi$ to $t=2.75 \pi$ in increments of $0.025 \pi$ (from top down).
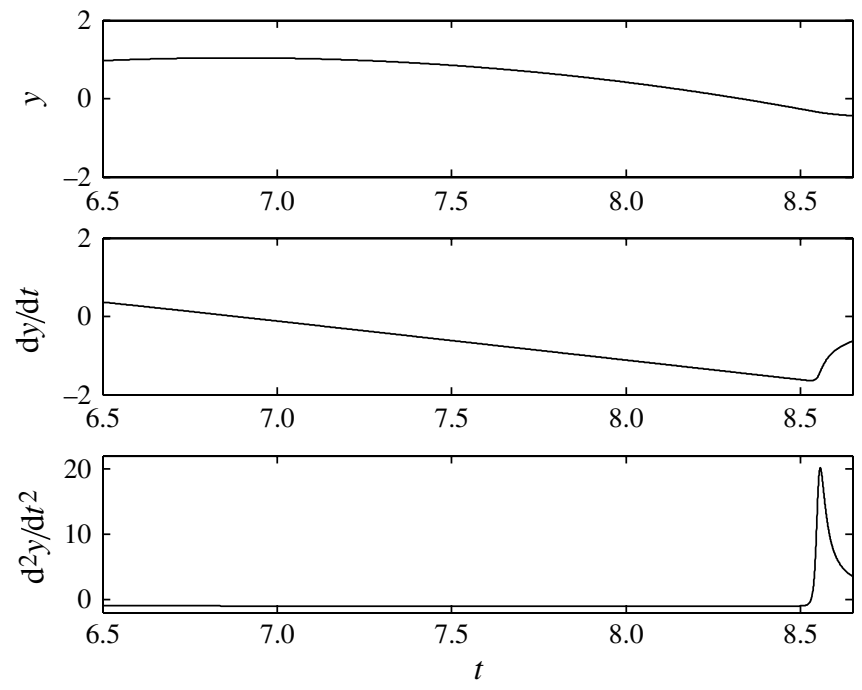

FIGURE 17. Vertical components of the location, velocity and acceleration of the Lagrangian marker at the centre of the peak $(x=0)$.

$x=\pi(p=\pi)$ where they appear to collide. This pattern is also clearly discernible in the wave profiles shown at the same times in figure 15.

While there are advantages to viewing the curvature as a function of the Lagrangian variable $p$, for example to observe the propagation of the curvature spikes with that of the wave profile, it is the curvature as a function of arclength that is important for assessing the nature of the singularity. The curvature profiles suggest the presence of two pole singularities in the complex arclength plane. If this is so, then the form fit (A 3) is not valid. Because of the symmetry in the profiles, if there are two 

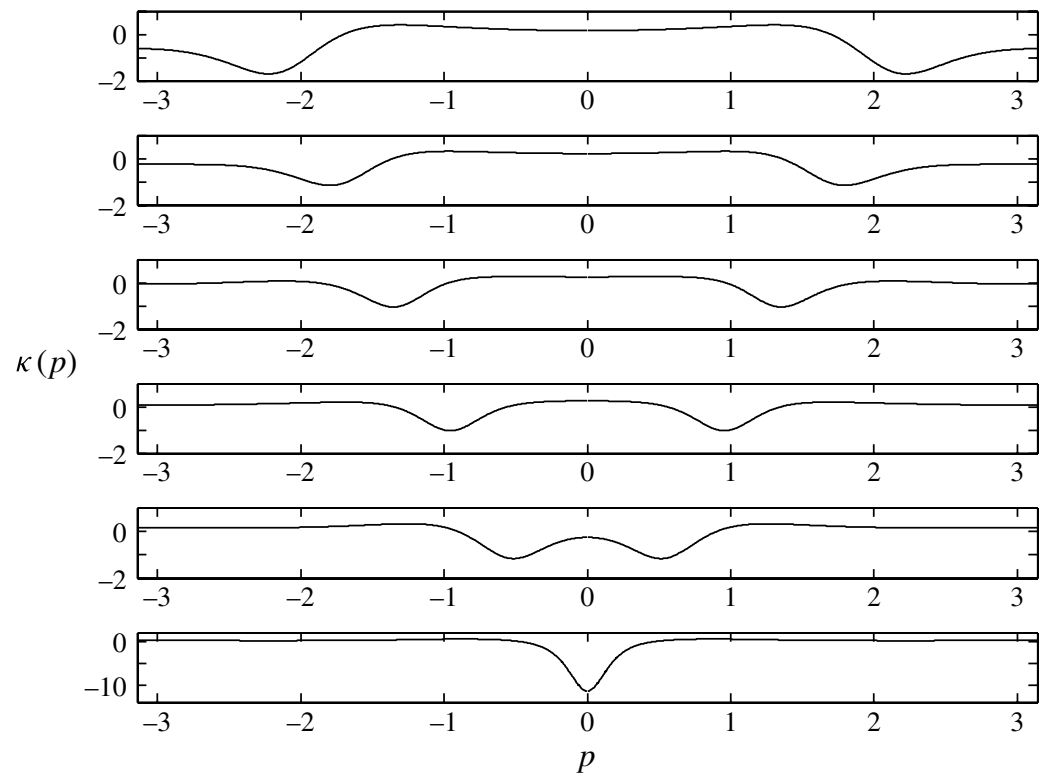

FIGURE 18. Successive curvature profiles starting from $t=0.75 \pi$ until $t=2 \pi$ in increments of $0.25 \pi$.

singularities they must be identical and lie at $s_{0}=\gamma+\mathrm{i} \delta$ and $s_{0}=(2 \pi-\gamma)+\mathrm{i} \delta$. By adding their contributions to the Fourier coefficients, the tail of the spectrum has the form

$$
\kappa_{k} \sim C \exp (-k \delta) \cos (\gamma k+\psi),
$$

where the assumption has been made that they are pole singularities. A similar approach was adopted by Baker et al. (1993), where the authors considered a pair of singularities placed symmetrically about the falling spike during Rayleigh-Taylor instability of two fluids. Formula (4.2) is then used to fit the curvature spectrum of $\kappa(s)$. When the singularities are close together, in other words $\gamma$ is small, then the slow variation in the cosine term in (4.2) makes it challenging to use a sliding fit that uses just a few points. Instead, a nonlinear least-square fitting procedure based on a robust version of the Levenberg-Marquardt algorithm (see Galassi et al. 2009) is applied to find the parameters $C, \delta, \gamma$ and $\psi$. At each time, a local least-squares fit based on data at $k, k+1, \ldots, k+L$ is performed. By studying the results of the fit as $k$ increases it is possible to confirm consistency in the assumptions of the fit and to pick the best values for the parameters in the fit. This procedure is performed only when there is evidence that there are two singularities, and that occurs from about $t \approx 2$ until $t \approx 6$.

The trajectories of the singularities are shown in figure 19. The singularities collide on the imaginary axis, a phenomenon already observed by Cowley et al. (1999) in singularity behaviour of vortex sheets. The most likely consequence of the collision is that the two pole singularities move along the imaginary axis, but at different locations. The standard sliding fit (A 3) to the Fourier spectra captures the singularity that is closest and the result is included in figure 19 as the vertical line down from the point of collision. The peak is collapsing during the close approach of the pole singularity along the imaginary axis towards the real axis. 


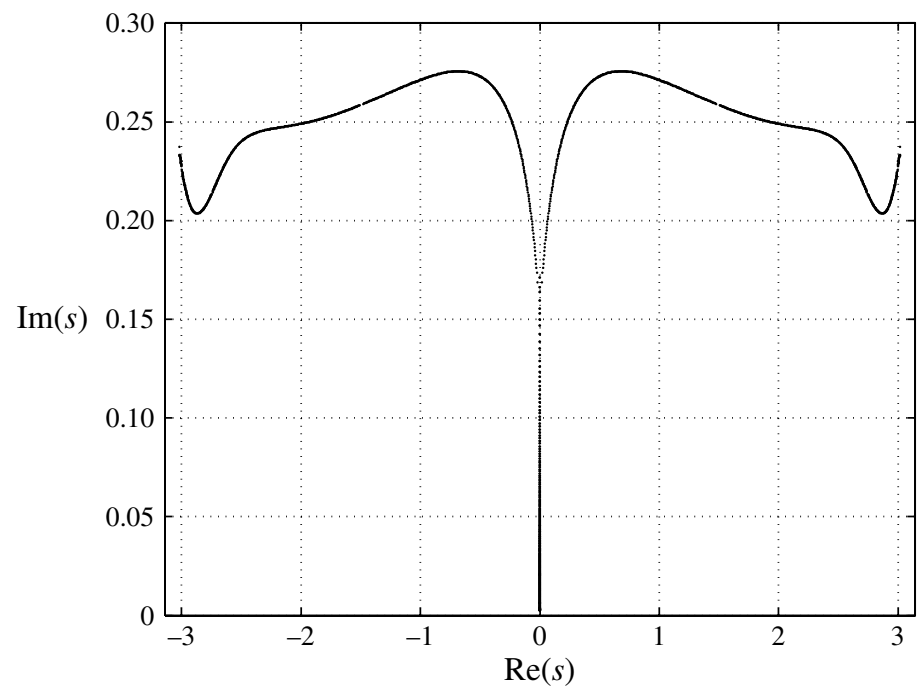

FIGURE 19. Trajectory of the pole singularities of the curvature in the complex arclength plane.

As a quick aside, the positions of the pole singularities coincide with the horizontal location of the curvature spikes. Otherwise, it is the close approach of the pole singularity to the real axis for late times that is relevant in assessing whether a curvature singularity forms on the wave profile in finite time. In figure 20, the distance $\delta$ of the pole singularity is shown as a function of time. For $\varepsilon=0.5$, the case considered so far for standing waves, $\delta$ approaches very closely to the real axis but very slowly, until the moment is reached when the falling peak in the wave profile suddenly smooths out and is absorbed into the wave. The moment, just past $t=8.5$, when the pole singularity races away from the real axis coincides with the time shown in figure 17 when the collapsing peak suddenly decelerates and this is the time at which the wave profile flattens out.

Also shown in figure 20 are the distances $\delta$ corresponding to other choices for $\varepsilon$. When $\varepsilon$ is small, the pole singularities move up and down the imaginary axis (or the line of symmetry corresponding to $x=\pi$ ) as the wave cycles through troughs and peaks. At large enough amplitudes, another pole singularity, presumably on the imaginary axis (or the line of symmetry corresponding to $x=\pi$ ) and above the one closest to the real axis, moves down fast enough to collide with the nearest singularity, and they separate symmetrically about the imaginary axis (or line of symmetry corresponding to $x=\pi$ ). These singularities can subsequently move together again, collide and move along the imaginary axis (or line of symmetry corresponding to $x=\pi$ ). In essence, these pole singularities move up and down, collide and move between lines of symmetry if the amplitudes are large enough.

\section{Conclusions}

Numerical simulation of water waves confirms the prediction from the work of Tanveer (1991) and Tanveer (1993) that there are pole singularities in the complex arclength plane of the curvature. These singularities move about the plane while retaining their form. Under the right conditions, typically large-amplitude waves, these 


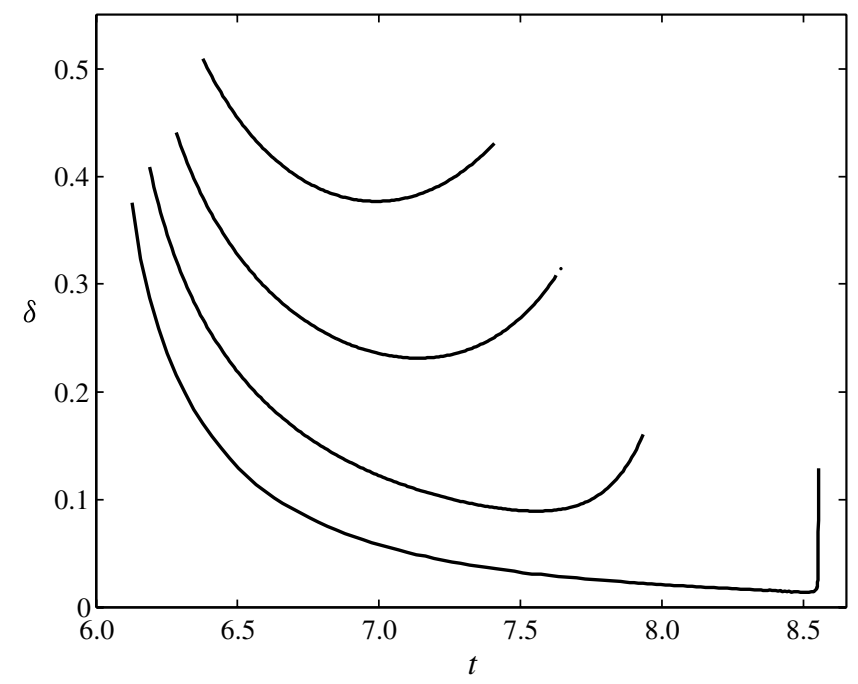

FIGURE 20. The distance $\delta$ of the pole singularities of the curvature from the real axis of the complex arclength plane for various $\varepsilon$ : from the top down, $\varepsilon=0.35,0.40,0.45,0.5$.

singularities approach the real axis very closely, resulting in the formation of very sharply peaked crests or tips of breaking waves. While the search has not been exhaustive, there is no evidence of the formation of curvature singularities on the wave profile in finite time. The study has used initial conditions that are analytic in a Lagrangian variable $p$, although this does not exclude the possibility of pole singularities in the curvature resulting from zeros in $s_{p}$ from being present initially.

Another singularity of importance is that associated with the profile $y(x)$. There are square-root singularities in the complex $x$ plane that move about while retaining their form. These singularities can and do reach the real axis in finite time, a time at which the profile has a vertical slope and is a signal that the wave is breaking. Even when waves do not break, the presence of these singularities affects the tail of the spectrum of $y(x)$ strongly. Indeed, this effect has been used to identify their presence numerically. Said differently, these singularities capture the full effects of nonlinearity. They impart accurately the exponentially small effects of nonlinear interactions.

We are greatly indebted to Professor S. Tanveer for many insightful discussions and help with the understanding of pole singularities in the complex arclength plane of the curvature. This work was support in part by the National Science Foundation through grant number OCE-0620885. Some of the computing was performed at the Ohio Supercomputer Centre, and we gratefully acknowledge the resources we received.

\section{Appendix A. Singularity detection}

Sulem et al. (1983) introduced a method to detect the presence of isolated singularities of a real-valued function in the complex plane by a form fit of its Fourier spectrum. Consider a function $u(z)$ which is analytic except at isolated singularities $z_{s}=\gamma+\mathrm{i} \delta$, in the neighbourhood of which it may be represented as

$$
u(z) \sim\left(z-z_{s}\right)^{\mu},
$$


where $\mu$ need not be real. Using the definition in Carrier, Krook \& Pearson (1966), the Fourier coefficients are

$$
u_{k}=\frac{1}{2 \pi} \int u(z) \mathrm{e}^{\mathrm{i} k z} \mathrm{~d} z .
$$

For real functions, $u_{-k}=u_{k}^{*}$. The asymptotic behaviour of the Fourier spectrum for $k \rightarrow+\infty$ is determined by the singularity in the upper half-plane which is closest to the real axis. Assume $z_{s}$ is the closest one to the real axis. Then as $k \rightarrow+\infty$, the Fourier coefficients have the asymptotic expansion

$$
u_{k} \sim C|k|^{-\mu-1} \exp (-k \delta+\mathrm{i} k \gamma)+D|k|^{-\mu-2} \exp (-k \delta+\mathrm{i} k \gamma)+\cdots .
$$

A derivation is available in Carrier et al. (1966).

If the singularity lies below the real axis, then a similar asymptotic representation applies to $k \rightarrow-\infty$. Fortunately, if $u(z)$ is real along the real axis, then the singularities must appear as complex conjugates, in which case only the singularity in the upper half-plane needs to be located.

The idea of tracking the singularity is to estimate the power of the singularity $\mu$ and its location $z_{s}$ by matching the numerically generated Fourier spectrum with the form (A 3). Provided the match is reliable, the coefficients $\mu$ and $z_{s}$ may be used to track the singularity in time.

The asymptotic expression (A 3) leads to two forms depending on whether the correction term involving $D$ is included:

$$
\begin{gathered}
\ln \left|u_{k}\right| \approx \ln C-(\mu+1) \ln |k|-\delta|k|, \\
\ln \left|u_{k}\right| \approx \ln C-(\mu+1) \ln |k|-\delta|k|+\frac{D}{k} .
\end{gathered}
$$

There are two ways to match the form (A 4) or (A 5) to the Fourier coefficients $u_{k}$ : a least-square method and a sliding fit method described next. For (A 4) use the equation at $k-1, k$ and $k+1$ to obtain a linear system which leads to exact solutions for $\ln C, \mu$ and $\delta$. Consider the results as valid for the choice of $k$. This process is then repeated consecutively over a range of $k$. For (A 5) use the equations at $k-1, k, k+1$ and $k+2$ to solve for the coefficients. In either case, the expectation is that as $k$ is increased, the solutions obtained from the linear systems converge to the true value of the parameters in the asymptotic expression (A 3).

Sometimes it is difficult to obtain satisfactory results, perhaps because of an oscillatory spectrum due to multiple singularities of comparable distances to the real axis or simply because of a lack of enough digits of accuracy in the Fourier spectrum. This difficulty may be offset by considering $q$ equations $(q \geqslant 3$ by using (A 4$)$ and $q \geqslant 4$ by using (A 5)) as a set of equations and obtaining the least-square solutions for the unknowns. For example, Sulem et al. (1983) used least squares in their original paper.

The horizontal location $\gamma$ of the singularity is found in a similar yet simpler way. The singularities appear periodically, since the function $u$ is $2 \pi$-periodic, so $\gamma$ may be understood as the value within $[0,2 \pi]$. The quantity $W_{k}=u_{k}|k|^{\mu+1} \exp (k \delta)$ is calculated and it follows that $W_{k} \sim C \exp (\mathrm{i} k \gamma)$, a purely oscillatory quantity, from which $\gamma$ may be determined.

A final note of caution. For the form fit to be reliable, enough of the tail of the spectrum must be available and accurate. If the singularity is far from the real axis, the spectrum decays very rapidly and not enough of the tail is available. If the singularity is very close to the real axis, it can be very difficult to ensure sufficient accuracy in a 
slowly decaying spectrum. These two factors limit the range in which singularities can be tracked. Furthermore, the form fit captures only the nearest singularity to the real axis. If another is of comparable distance, the asymptotic form (A 3) is not valid and the form fit based on (A 4) and (A 5) will fail.

\section{Appendix B. Approximate solution for a plunging tip}

A family of solutions that take the form of hyperbolae in free fall has been proposed as candidates for the tip of a plunging breaker by Longuet-Higgins (1972, 1980). The solutions are exact, but should be viewed as the leading-order contribution to a local expansion near the tip.

The results can be expressed in terms of Lagrangian motion which is more convenient for the study reported here. Specifically, the location of the free surface, expressed as a complex function, is written in parametric form,

$$
\begin{aligned}
z(p, t) & =\frac{a+\mathrm{i} b}{2} \mathrm{e}^{p+f+\mathrm{i} \delta}-\frac{a-\mathrm{i} b}{2} \mathrm{e}^{-p-f+\mathrm{i} \delta}-\frac{g t^{2}}{2} \\
& =[a \sinh (p+f)+\mathrm{i} b \cosh (p+f)] \mathrm{e}^{\mathrm{i} \delta}-\frac{g t^{2}}{2},
\end{aligned}
$$

where $a, b, f$ and $\delta$ are real-valued functions of time. The form (B 1) represents a hyperbola rotated by $\delta$ from the vertical position, with upward arms in a frame accelerating downwards with acceleration $g$.

The complex velocity potential is written as

$$
\Phi(z, t)=\frac{\alpha}{2} \mathrm{e}^{\mathrm{i} \sigma} z^{2}-g t y,
$$

where $\alpha$ and $\sigma$ are real-valued functions of time. By evaluating this potential on the free surface (B 1) and substituting into the equation of motion (2.3), an exact solution is achieved with the balance of exponential terms,

$$
\begin{aligned}
& \frac{\mathrm{d} \rho}{\mathrm{d} t}+\rho \frac{\mathrm{d} f}{\mathrm{~d} t}=\alpha \rho \cos [\sigma+2(\delta+\psi)], \\
& \frac{\mathrm{d} \rho}{\mathrm{d} t}-\rho \frac{\mathrm{d} f}{\mathrm{~d} t}=\alpha \rho \cos [\sigma+2(\delta-\psi)], \\
& \frac{\mathrm{d} \delta}{\mathrm{d} t}+\frac{\mathrm{d} \psi}{\mathrm{d} t}=-\alpha \sin [\sigma+2(\delta+\psi)], \\
& \frac{\mathrm{d} \delta}{\mathrm{d} t}-\frac{\mathrm{d} \psi}{\mathrm{d} t}=-\alpha \sin [\sigma+2(\delta-\psi)] .
\end{aligned}
$$

Here the change of variables $a+\mathrm{i} b=2 \rho \exp (\mathrm{i} \psi)$ has been used. Note that $\tan (\psi)=b / a$ gives the slope of the asymptotes for the hyperbola in the rotated frame.

A solution to Bernoulli's equation can be obtained by substituting (B 1) and (B 2) and balancing exponentials. There are also terms independent of $p$ that effectively determine the time-dependent constant in Bernoulli's equation. An exact solution occurs when

$$
\begin{gathered}
\frac{\mathrm{d}}{\mathrm{d} t}\left[\alpha \rho^{2} \mathrm{e}^{2 f} \cos (\sigma+2(\delta+\psi))\right]=\alpha^{2} \rho^{2} \mathrm{e}^{2 f}, \\
\frac{\mathrm{d}}{\mathrm{d} t}\left[\alpha \rho^{2} \mathrm{e}^{-2 f} \cos (\sigma+2(\delta-\psi))\right]=\alpha^{2} \rho^{2} \mathrm{e}^{-2 f}
\end{gathered}
$$


With the definition $2 \theta=\sigma+2 \delta$, (B 3)-(B 8) can be written as a first-order system,

$$
\begin{gathered}
\frac{\mathrm{d} \alpha}{\mathrm{d} t} \cos (2 \psi)=-\alpha^{2} \cos (2 \theta) \\
\frac{\mathrm{d} \theta}{\mathrm{d} t} \cos (2 \psi)=-\alpha \sin (2 \theta) \cos (4 \psi) \\
\frac{\mathrm{d} \psi}{\mathrm{d} t}=-\alpha \cos (2 \theta) \sin (2 \psi) \\
\frac{\mathrm{d} \rho}{\mathrm{d} t}=\alpha \rho \cos (2 \theta) \cos (2 \psi) \\
\frac{\mathrm{d} \delta}{\mathrm{d} t}=-\alpha \sin (2 \theta) \cos (2 \psi) \\
\frac{\mathrm{d} f}{\mathrm{~d} t}=-\alpha \sin (2 \theta) \sin (2 \psi)
\end{gathered}
$$

Note that the first three equations form a closed system for $\alpha, \theta$ and $\psi$, and the last three equation determine $\rho, \delta$ and $f$.

Unfortunately, there is no closed-form solution to these equations, but the long time behaviour of a falling tip can be determined under the assumptions that the slope of the asymptote for the hyperbola is large (the tip is sharp), $2 \psi=\pi-2 \epsilon$. The half-angle between the asymptotes that include the hyperbola is $\epsilon$. The first two equations in the system, (B 9) and (B 10), simplify,

$$
\begin{aligned}
& \frac{\mathrm{d} \alpha}{\mathrm{d} t}=\alpha^{2} \cos (2 \theta), \\
& \frac{\mathrm{d} \theta}{\mathrm{d} t}=\frac{\alpha}{2} \sin (2 \theta) .
\end{aligned}
$$

The curves $\alpha=C \sin (2 \theta)$ form the family of trajectories in the phase plane for $\alpha, \theta$. The solutions of interest are those approaching the critical point at the origin of the phase plane with $\alpha, \theta<0$. With $|\theta| \ll 1$, (B 15) has the approximate solution

$$
\alpha=-\frac{C_{1}}{1+C_{1} t},
$$

where the origin in time is chosen when the plunging tip of the wave is already formed and thin, and $\alpha(0)=-C_{1}$. The rest of the equations in the system are easily solved. In particular,

$$
\rho=C_{2}\left(C_{1} t+1\right), \quad \epsilon=\frac{C_{3}}{\left(C_{1} t+1\right)^{2}},
$$

which leads to

$$
a=\frac{C_{3}}{C_{1} t+1}, \quad b=2 C_{2}\left(C_{1} t+1\right) .
$$

These results are consistent with the results obtained before (Longuet-Higgins 1980), where it is shown that the appropriate choice for the hyperbolae can match plunging tips very well.

\section{REFERENCES}

BAKER, G. R. 1983 Generalized vortex methods for free-surface flows. In Waves on Fluid Interfaces, pp. 53-81. Academic. 
Baker, G. R., CAflisch, R. E. \& Siegel, M. 1993 Singularity formation during Rayleigh-Taylor instability. J. Fluid Mech. 252, 51-78.

BAKer, G., MCCrory, R., Verdon, C. \& OrszaG, S. 1987 Rayleigh-Taylor instability of fluid layers. J. Fluid Mech. 178, 161-178.

Baker, G. R., Meiron, D. I. \& Orszag, S. A. 1980 Vortex simulations of the Rayleigh-Taylor instability. Phys. Fluids 23, 1485-1490.

Baker, G. R., Meiron, D. I. \& Orszag, S. A. 1982 Generalized vortex methods for free-surface flow problems. J. Fluid Mech. 123, 477-501.

BAKer, G. \& MoORE, D. 1989 The rise and distortion of a two-dimensional gas bubble in an inviscid liquid. Phys. Fluids A 1, 1451-1459.

BAKER, G. R. \& NACHBIN, A. 1998 Stable methods for vortex sheet motion in the presence of surface tension. SIAM J. Sci. Comput. 19, 1737-1766.

Caflisch, R. E. \& ORellana, O. F. 1989 Singular solutions and ill-posedness for the evolution of vortex sheets. SIAM J. Math. Anal. 20, 293-307.

CAFlisCH, R. \& SEMMES, S. 1990 A nonlinear approximation for vortex sheet evolution and singularity formation. Physica D 41, 197-207.

Carrier, G. F., Krook, M. \& Pearson, C. E. 1966 Functions of a Complex Variable: Theory and Technique. McGraw-Hill.

Ceniceros, H. D. \& Hou, T. Y. 1998 Convergence of a non-stiff boundary integral method for interfacial flows with surface tension. Maths Comput. 67, 137-182.

Cowley, S. J., BAKer, G. R. \& TANVeER, S. A. 1999 On the formation of Moore curvature singularities in vortex sheets. J. Fluid Mech. 378, 233-267.

Craig, W. \& Wayne, C. E. 2007 Mathematical aspects of surface water waves. Russ. Math. Surv. 62, 453-473.

Ely, J. S. \& BAKer, G. R. 1993 High-precision calculations of vortex sheet motion. J. Comput. Phys. 111, 275-281.

Fontelos, A. \& DE LA Hoz, F. 2010 Singularities in Water Waves and the Rayleigh-Taylor problem. J. Fluid Mech. 651, 211-239.

Galassi, M., Davies, J., Theiler, J., Gough, B., Jungman, G., Alken, P., Booth, M. \& Rossi, F. 2009 Nonlinear least-squares fitting. In GNU Scientific Library Reference Manual, pp. $403-414$.

Germain, P., Masmoudi, N. \& Shatah, J. 2009 Global solutions for the gravity water waves equation in dimension 3. C. R. Acad. Sci. Paris (I) 346, 897-902.

Gottlieb, D. \& Orszag, S. A. 1977 Numerical Analysis of Spectral Methods: Theory and Applications, CBMS-NSF Regional Conference Series in Applied Mathematics. SIAM.

Hou, T. Y. \& HU, G. 2003 Singularity formation in three-dimensional vortex sheets. Phys. Fluids 15, 147-172.

Hou, T. Y., Lowengrub, J. S. \& Shelley, M. J. 1994 Removing the stiffness from interfacial flows with surface tension. J. Comput. Phys. 114, 312-338.

IshiHARA, T. \& KANEDA, Y. 1994 Spontaneous singularity formation in the shape of vortex sheet in three-dimensional flow. J. Phys. Soc. Japan 63, 388-392.

KANO, T. \& NishidA, T. 1979 Sur les ondes de surface de l'eau avec une justification mathématique des equations des ondes en eau peu profonde. J. Maths Kyoto Univ. 19, 335-370.

KRASNY, R. 1986 A study of singularity formation in a vortex sheet by the point-vortex approximation. J. Fluid Mech. 167, 65-93.

Kuznetsov, E. A., Spector, M. D. \& Zakharov, V. E. 1994 Formation of singularities on the free surface of an ideal fluid. Phys. Rev. E 49 (2), 1283-1290.

Longuet-Higgins, M. 1972 A class of exact, time-dependent, free-surface flows. J. Fluid Mech. 55, 529-543.

Longuet-Higgins, M. 1980 On the forming of sharp corners at a free surface. Proc. R. Soc. Lond. A 371, 453-478.

Longuet-Higgins, M. 1982 Parametric solutions for breaking waves. J. Fluid Mech. 121, 403-424. 
Longuet-Higgins, M. \& Dommermuth, D. 2001 On the breaking of standing waves by falling jets. Phys. Fluids 13, 1652-1659.

Mercer, G. N. \& Roberts, A. J. 1992 Standing waves in deep water. Their stability and extreme form. Phys. Fluids A 4, 259-269.

Moore, D. W. 1979 The spontaneous appearance of a singularity in the shape of an evolving vortex sheet. Proc. R. Soc. Lond. A 365, 105-119.

NIE, Q. \& BAKER, G. 1998 Application of adaptive quadrature to axi-symmetric vortex sheet motion. J. Comput. Phys. 143, 49-69.

OKAMURA, M. 1998 On the enclosed crest angle of the limiting profile of standing waves. Wave Motion 28, 79-87.

Penny, W. G. \& Price, A. T. 1952 Finite periodic stationary gravity waves in a perfect fluid. Phil. Trans. R. Soc. Lond. 244, 254-284.

SHELlEY, M. J. 1992 A study of singularity formation in vortex-sheet motion by a spectrally accurate vortex method. J. Fluid Mech. 244, 493-526.

Shinbrot, M. 1976 The initial value problem for surface waves under gravity. Indiana Univ. Math. J. 25, 281-300.

Sulem, C., Sulem, P.-L. \& FRISCH, H. 1983 Tracing complex singularities with spectral methods. J. Comput. Phys. 50, 138-161.

TAnveer, S. A. 1991 Singularities in water waves and Rayleigh-Taylor instability. Proc. R. Soc. Lond. A 435, 137-158.

TANVEER, S. A. 1993 Singularities in the classical Rayleigh-Taylor flow: formation and subsequent motion. Proc. R. Soc. Lond. A 441, 501-525.

Toland, J. 1996 Stokes waves. Topol. Meth. Nonlinear Anal. 7, 1-48.

Vichnevetsky, R. \& Bowles, J. B. 1982 Fourier Analysis of Numerical Approximations of Hyperbolic Equations. SIAM Studies in Applied Mathematics.

WU, S. 1997 Well-posedness in Sobolev spaces of the full water wave problem in 2-D. Invent. Math. 130, 39-72.

WU, S. 1999 Well-posedness in Sobolev spaces of the full water wave problem in 3-D. J. Am. Math. Soc. 12, 445-495.

WU, S. 2009 Almost global wellposedness of the 2-D full water water problem. Invent. Math. 177, 45-135. 